\title{
1 Manipulation of carotenoid metabolism stimulates biomass and stress tolerance in tomato
}

2 Jose G. Vallarino ${ }^{1}$, Jianing $\mathrm{Mi}^{2}$, Ivan Petř́ik ${ }^{3}$, Ondřej Novák ${ }^{3}$, Sandra M. Correa ${ }^{1}$, Monika

3 Kosmacz ${ }^{1,2}$, Michel Havaux ${ }^{4}$, Manuel Rodriguez-Concepcion 5, Salim Al-Babili², Alisdair R.

4 Fernie $^{1}$, Aleksandra Skirycz ${ }^{1,6}$, Juan C. Moreno ${ }^{1,2 *}$

$5 \quad{ }^{1}$ Max Planck Institut für Molekulare Pflanzenphysiologie, Am Mühlenberg1 D-14476, Potsdam6 Golm, Germany.

$7 \quad{ }^{2}$ Center for Desert Agriculture, Biological and Environmental Science and Engineering Division 8 (BESE), King Abdullah University of Science and Technology (KAUST), Thuwal, Saudi Arabia.

$9{ }^{3}$ Laboratory of Growth Regulators, Faculty of Science, Palacký University and Institute of 10 Experimental Botany, The Czech Academy of Sciences, Šlechtitelů 27, CZ-78371 Olomouc, Czech 11 Republic.

$12{ }^{4}$ Aix-Marseille University, CEA, CNRS UMR7265, BIAM, CEA/Cadarache, F-13108 Saint-Paul13 lez-Durance, France.

$14{ }^{5}$ Institute for Plant Molecular and Cell Biology (IBMCP) UPV-CSIC, 46022 Valencia, Spain.

$15{ }^{6}$ Boyce Thompson Institute, Cornell University, Ithaca, NY, United States.

*Corresponding author. Email: juancamilo.morenobeltran@KAUST.edu.sa

Running title: Carotenoid metabolism influences biomass and stress tolerance

31 The author(s) responsible for distribution of materials integral to the findings presented in this is (are): Juan C. Moreno (juancamilo.morenobeltran@KAUST.edu.sa) 


\section{ABSTRACT}

36 Improving yield, nutritional value and tolerance to abiotic stress are major targets of current

37 breeding and biotechnological approaches that aim at increasing crop production and ensuring food

38 security. Metabolic engineering of carotenoids, the precursor of Vitamin-A and plant hormones that

39 regulate plant growth and response to adverse growth conditions, has been mainly focusing on

40 provitamin A biofortification or the production of high-value carotenoids. Here, we show that the

41 introduction of a single gene of the carotenoid biosynthetic pathway in different tomato cultivars

42 simultaneously improved photosynthetic capacity and tolerance to various abiotic stresses (e.g.,

43 high light, salt, and drought), caused an up to $77 \%$ fruit yield increase and enhanced fruit's

44 provitamin A content and shelf life. Our findings pave the way for developing a new generation of

45 crops that combine high productivity and increased nutritional value with the capability to cope

46 with climate change-related environmental challenges.

47

48 Keywords: abiotic stress tolerance, apocarotenoids, biomass and yield, $\beta$-carotene, carotenoids, 49 metabolites and lipids, photoprotection, phytohormones, plant architecture, xanthophylls. 
52 Climate change and the increasing world population are serious challenges facing world agriculture

53 (Pareek et al., 2020). Indeed, current estimates indicate that food production should be doubled by

542050 (Ort et al., 2015; Xu, 2016). However, global warming and the anthropogenic activities that

55 affect agricultural ecosystems and subsequent crop yield render this doubling a very difficult goal to

56 achieve. Moreover, abiotic stresses, and especially salinity and drought, cause considerable crop

57 losses, with yield reductions of almost 50\% (Hussain et al., 2019; Roy et al., 2014). Therefore, a

58 new generation of crops with enhanced fitness - as exemplified, for instance, by simultaneously

59 improved photosynthetic efficiency, stress tolerance, and yield - are urgently needed to meet the

60 desired levels of crop productivity. In the past decade, photosynthesis and photorespiration have

61 been the preferred targets for manipulation to improve plant yield (Ding et al., 2016; Lopez-

62 Calcagno et al., 2019; Simkin et al., 2017; Simkin et al., 2015; South et al., 2019; Timm et al.,

63 2015). For example, two breakthrough genetic strategies for manipulating the xanthophyll cycle

64 (manipulation of three genes) and glycolate metabolism (introduction of five genes) have

65 documented increases in plant biomass of between $15 \%$ and $37 \%$, respectively, in the cash crop

66 tobacco (Kromdijk et al., 2016; South et al., 2019). However, to date, neither of these strategies

67 have been demonstrated to work in food crops. Moreover, similar manipulation of the xanthophyll

68 cycle in Arabidopsis resulted in a contradictory reduction in plant biomass (Garcia-Molina and

69 Leister, 2020), bringing into question the general applicability of this method.

Another possibility for manipulating plant yield and fitness in crops might be provided by the carotenoids (e.g., $\beta$-carotene), which are isoprenoid pigments that rank among the most important plant secondary metabolites due to the diverse functions they fulfil in photosynthesis and signaling. Within chloroplasts, carotenoids like $\beta$-carotene and xanthophylls are key components of photosynthetic membranes and form pigment-protein complexes that are essential for photoprotection (Niyogi and Truong, 2013; Xu et al., 2020). $\beta$-carotene is also the precursor of abscisic acid (ABA) and strigolactones (SLs), so alterations in carotenoid content can affect hormone content and subsequent plant development and physiology (Al-Babili and Bouwmeester, 2015; Nambara and Marion-Poll, 2005). In recent years, new signaling and growth-promoting functions have been reported for carotenoid-derived molecules (commonly referred to as apocarotenoids), including $\beta$-cyclocitral $(\beta-\mathrm{cc})$, dihydroactinidiolide (dhA), and zaxinone (Zax) (D'Alessandro et al., 2018; D'Alessandro et al., 2019; Dickinson et al., 2019; Hou et al., 2016; Wang et al., 2019). In animals, carotenoids consumed in the diet are also cleaved to produce retinoids (including vitamin A) and other molecules with signaling and health-promoting properties 
84 (Rodriguez-Concepcion et al., 2018). $\beta$-carotene is the main precursor of vitamin A in animals and

85 the main precursor of several apocarotenoids and plant hormones in plants; therefore, increased

86 accumulation of $\beta$-carotene might indirectly influence plant growth and development, as well as

87 improve the nutritional value. $\beta$-carotene is produced by the action of lycopene $\beta$-cyclase (LCYB),

88 indicating a potential for genetic manipulation of the expression of this gene as a two-for-one

89 solution to improve both the fitness and the nutritional value of the chosen crop.

90 In our previous work, we expressed the LCYB-encoding DcLCYB1 gene from carrot (Daucus

91 carota) in tobacco and demonstrated growth-promoting and developmental effects of this gene

92 (Moreno et al., 2020). Interestingly, these tobacco lines also showed enhanced tolerance to abiotic

93 stresses, in addition to enhancement of biomass, yield, and photosynthetic efficiency (Moreno et al.,

94 2021). These beneficial effects were mainly triggered by an enhanced accumulation of the

95 phytohormones $\mathrm{ABA}$ and gibberellic acid (GA), but they were also a result of the greater

96 photoprotection afforded by the accumulation of xanthophylls. We therefore hypothesized that any

97 LCYB-encoding gene, independent of its origin (plant or bacterial), might trigger similar beneficial

98 effects to those observed with the carrot DcLCYB1 gene in tobacco (Moreno et al., 2020).

99 In the present study, we explored this hypothesis using previously generated tomato cultivars that 100 overexpress three different $L C Y B$ genes (from plant and bacterial origins) following plastid or 101 nuclear transformation. We confirmed that the overexpression of any $L C Y B$ gene is sufficient to 102 trigger a molecular response that results in modulation of carotenoid (pro-vitamin A) and hormone 103 content, with a subsequent alteration in plant architecture, photosynthetic efficiency, stress 104 tolerance, and yield. 


\section{RESULTS}

\section{Tomato productivity under different environmental conditions}

Given our recent findings that expression of the carrot $D c L C Y B 1$ gene resulted in increased photosynthetic efficiency, photoprotection, stress tolerance, plant biomass, and yield in tobacco (Moreno et al., 2021; Moreno et al., 2020), we decided to evaluate whether manipulation of LCYB activity could confer similar growth advantages in an economically important food crop. We tested our hypothesis by exploiting the availability of several tomato cultivars overexpressing different LCYB-encoding genes. In particular, we used a Red Setter cultivar with a nuclear construct overexpressing a tomato LCYB (line H.C.) and two transplastomic lines expressing LCYBencoding genes from daffodil in the IPA6+ background (line pNLyc\#2) or from the bacterium Erwinia uredovora (renamed Pantoea ananatis) in the IPA6- background (line LCe) (see Materials and Methods; Table S1). Growth evaluation under different climate conditions (fully controlled, semi-controlled, and uncontrolled conditions) revealed robust and homogeneous changes in plant height (increased and reduced plant height for transplastomic and nucear lines, respectively) of the transgenic lines in comparison to their respective wild type in all climate conditions (fig. S1). Due to the robustness of the phenotypes, we selected the semi-controlled conditions (greenhouse) to perform a detailed molecular and physiological characterization of this phenomenon. Interestingly, the pNLyc\#2 and LCe transplastomic lines showed longer stems than their respective wild-type plants, thereby allowing a more spaced allocation of their leaves along the stem. By contrast, the H.C. nuclear line showed reduced plant height (Fig. 1A-C). In addition, leaves from pNLyc\#2 were larger than the IPA6+ leaves, while leaves from the H.C. line were smaller than those from its wild type R.S. (fig. S2A, D). By contrast, leaves from the LCe line showed sizes similar to the wild type (fig. S2G). The fruit size was similar to the wild type in the pNLyc\#2 line but was slightly larger in the LCe line (fig. S2J), while the fruit from the H.C. line were considerably larger when compared to those from its respective wild type (fig. S2B, E, H, J).

Plant biomass was assessed in all the lines to determine plant productivity. Interestingly, the different $L C Y B$ transgenic lines showed different biomass partitioning when comparing leaves, stem, and fruit (Fig. 1M-O). For instance, the transplastomic pNLyc\#2 showed a clear increase in plant height $(\sim 30 \%)$ and stem biomass (45\%), but no changes in leaf biomass or leaf number (Fig. 1J, K M, N). In addition, fruit biomass (37\%) and fruit number were reduced, although the fruit size observed in pNLyc\#2 was similar to the wild type (Fig. 1L). By contrast, the H.C. line showed reduced plant height (40\%) and stem biomass (30\%), but no changes in leaf biomass (Fig. 1J, M, N). Interestingly, the H.C. line displayed a reduced number of leaves compared to the wild type 
139

140

141

142

143

144

145

146

147

148

149

150

151

152

153

154

155

156

157

158

159

160

161

162

163

164

165

166

167

168

169

170

(Fig. 1K). In addition, the H.C. fruit biomass was increased by $77 \%$ compared to the wild type R.S. (Fig. 10), in line with the increased fruit number and size displayed by this genotype (Fig. 1H, L). The LCe transplastomic line showed increased plant height ( 20\%) and leaf biomass (17\%), but no significant changes in stem biomass (Fig. 1J, M, N). Its fruit biomass was increased up to $45 \%$ relative to the wild type IPA6- (Fig. 10). In this line, the leaf and fruit number remained the same as in the wild type (Fig. 1K, L). Seed production in pNLyc\#2 and LCe transplastomic lines was lower than in their wild types, while H.C. seed production was approximately $1000 \%$ higher than in its respective wild type (Fig. 1P-R). Biomass quantification in plants grown under fully controlled and uncontrolled conditions showed similar patterns of biomass redistribution (as in the greenhouse) in the different plant tissues (figs. S3 -S4), but also revealed delayed and accelerated development for the pNLyc\#2 and H.C. lines, respectively, while the LCe line showed wild-typelike development (figs. S4-S5).

\section{$L C Y B$-overexpressing lines show different carotenoid profiles in leaves and fruit}

We sought further insights into the different biomass accumulation patterns in leaves and fruit in the transgenic lines by investigating carotenoid accumulation in both organs, since an altered carotenoid content might affect hormone content and, thereby, plant growth. Transgenic lines expressing plant $L C Y B$ s showed a reduction in total leaf carotenoid content, with strong decreases in lutein and a lesser decrease in neoxanthin, but strong increases in violaxanthin and zeaxanthin levels. In addition, the H.C. line displayed a slight reduction in $\beta$-carotene levels. By contrast, the total carotenoid content in the bacterial $L C Y B$-expressing LCe line remained essentially the same as in the wild type, with some slight reductions in $\beta$-carotene and zeaxanthin levels in the leaves (Fig. 2A and fig. S6A, C, E).

In the fruit, the total carotenoid content in the transplastomic pNLyc\#2 and LCe lines was unchanged, while the total carotenoid content was reduced in the H.C. line. Transgenic lines expressing plant $L C Y B$ s showed a strong accumulation of fruit $\beta$-carotene and strong reductions in lycopene, lutein, and phytoene, while an increase in $\beta$-carotene was only observed for the bacterial $L C Y B$-expressing LCe line (Fig. 2A and fig. S6B, D, F). In addition, carotenoid-rich crystal structures were observed by confocal microscopy in the fruit of the transgenic lines (fig. S7). Due to the possibility that other isoprenoid pathways might have been affected by $L C Y B$ expression (Moreno et al., 2020), we also determined the chlorophyll and tocopherol (vitamin E) content in the leaves and fruit (fig. S8). Chlorophyll contents remain unchanged in the pNLyc\#2 and H.C. lines 
171 (with the exception of a slight reduction in chlorophyll $b$ in the H.C. line), while $\gamma$ - and $\alpha$-tocopherol

172 contents were increased. The LCe line showed a reduction in $\alpha$-tocopherol (fig. S8). By contrast, the

173 tocopherol content ( $\alpha, \delta$, and $\gamma$-tocopherol) increased strongly in fruit of the pNLyc\#2 line, while

174 remaining unaltered in the H.C. and LCe lines (fig. S8).

175 Hormone metabolism is altered in $L C Y B$-overexpressing lines

176 Altered $\beta$-carotene accumulation might influence the content of $\beta$-carotene-derived and/or 177 isoprenoid-derived hormones (e.g., ABA and Gas, respectively), thereby influencing plant growth 178 and development. Therefore, we profiled the plant hormones to gain further insights into their 179 contribution to the observed growth phenotype. The lines were characterized by significant increases in $\mathrm{ABA}$ and jasmonic acid (JA) for pNLyc\#2; $\mathrm{ABA}$ reduction and $\mathrm{GA}_{1}$ and IAA increments for H.C.; and ABA and $\mathrm{GA}_{1}$ reductions and JA and JA-Ile increments in LCe in leaves (Fig. 2B). By contrast, stronger significant changes in hormone content were found in fruit. ABA, JA, and JA-Ile were increased, while indole acetic acid (IAA), the most bioactive auxin (Aux), was reduced in both the pNLyc\#2 and H.C. lines but increased in the LCe line (Fig. 2B). In addition, SA was increased only in the pNLyc\#2 line, whereas isopentenyladenine (iP), an active cytokinin (CK), was increased in the pNLyc\#2 and LCe lines (Fig. 2B). Phaseic acid, a bioactive ABA catabolite, showed increased and reduced contents in the pNLyc\#2 and H.C. lines, respectively. Intermediates of the ABA, GA, Aux, CKs, and JA metabolic pathways were also differentially affected in leaves and fruit (fig. S9-10).

\section{Effects of carotenoid accumulation on apocarotenoid metabolism in leaves and fruit}

$192 \beta$-carotene and xanthophylls are the main precursors of non-hydroxylated and hydroxylated 193 apocarotenoids, respectively. Growth-promoting and signaling properties of some apocarotenoids 194 (e.g., $\beta$-cyclocitral and zaxinone) have been reported in rice, tomato, and Arabidopsis (Dickinson et 195 al., 2019; Wang et al., 2019). These previous findings and the altered pigment content observed in 196 the leaves and fruit of the transgenic lines led us to profile apocarotenoid species in order to 197 determine their contribution to the observed phenotypes (Fig. 2C and fig. S11-15). In leaves, non198 hydroxylated apocarotenoids showed few increases or wild-type-like accumulation (fig. S12), in 199 line with the wild-type-like $\beta$-carotene content in the transgenic lines. By contrast, hydroxylated 200 apocarotenoids showed strong reductions due to a strong decrease in lutein content (fig. S13). The 201 non-hydroxylated apocarotenoids in fruit showed a strong and significant accumulation (due to 202 enhanced $\beta$-carotene content; fig. S14), while the hydroxylated apocarotenoids exhibited strong 
203

204

205

206

207

208

209

210

211

212

213

214

215

216

217

218

219

220

221

222

223

224

225

226

227

228

229

230

231

232

233

234

reductions due to the lower lutein content in the fruit (fig. S14). Growth regulators, such as $\beta$-cc and Zax, were mainly found at reduced levels in the leaves and fruit (Fig. 2C). Other apocarotenoids with biological activity, such as $\beta$-ionone, showed enhanced accumulation in the fruit (fig. S11).

Primary metabolites and lipid metabolism are altered in leaves and fruit of $L C Y B$-expressing lines

The strong changes in pigment, hormone, and apocarotenoid contents led us to investigate the impact of these changes on other metabolic pathways. GC-MS metabolite profiling showed significant changes in sucrose and its derivatives (e.g., fructose, galactinol, raffinose), glycolytic intermediates (e.g., glucose, G6P, Fru6P) and TCA cycle intermediates (e.g., malate and fumarate) in the leaves and fruit of the transgenic lines (Fig. 3B; fig. S16). These changes were reflected, for instance, in changes in G6P-derived compounds (e.g., trehalose, maltotriose, maltose, myo-inositol, and erythritol) and amino acids derived from glycerate (e.g., O-acetylserine [OAS]), pyruvate (e.g., valine, alanine, leucine), shikimate (e.g., phenylalanine and tryptophan), malate (e.g., aspartic acid, asparagine, $\beta$-alanine, and methionine), and 2-oxoglutarate (e.g., glutamic acid, glutamine, GABA, and ornithine) (Fig. 3B). In addition, due to the structural function of carotenoids ( $\beta$-carotene and xanthophylls) in membrane composition, together with lipids, we determined the lipid composition in leaves and fruit. Lipid profiling revealed no significant differences in the leaves, while marked significant differences were observed, mainly for structural lipids, in the fruit of pNLyc\#2 and H.C. lines (Fig. 3B; fig. S17). In the fruit of pNLyc\#2, a total of 17 galactolipids (GLs) (e.g., mono- and di-galactosyldiacylglycerol, [MGDG and DGDG, respectively]) and 32 phospholipids (PLs) (e.g., phosphatidylcholine [PC], phosphatidylethanolamine [PE], phosphatidylglycerol [PG], and phosphatidylserine [PS]) exhibited significant changes in their ratio abundances (Fig. 3B), with levels of nine GLs reduced and eight increased, while the trend for PLs differed, where abundance rations were reduced for seven PLs and increased for 25 PLs. The general trend for sulfolipids (SLs) (e.g., sulfoquinovosyl diacylglycerol [SQDG]) and di- and tri-acylglycerols (DAGs/TAGs) was a reduced abundance, with the exception of two SL species (Fig. 3B). By contrast, in the H.C. line, most of the lipid species that showed significantly different levels displayed a reduced ratio abundance, with a few exceptions (e.g., two DAGs, four TAGs, one PC, and two PEs) that showed increased content (Fig. 3B).

Photosynthetic parameters are influenced by carotenoid accumulation and plant architectural changes in tomato $L C Y B$-expressing lines 
235 The changes in plant growth and architecture induced by modifications in pigment and hormone

236 contents prompted subsequent analysis of several photosynthetic parameters. Photosynthetic

237 measurements were performed in tomato plants (49 days old) grown under greenhouse conditions

238 (fig. S18). $\mathrm{CO}_{2}$ assimilation was significantly increased for the H.C. line, relative to its wild type,

239 whereas the transplastomic lines were the same as their wild types (Fig. 4A). Despite some

240 unaltered photosynthetic parameters, the ФPSII, which reflects plant fitness, was increased in all the

241 lines (Fig. 4B). Interestingly, $\mathrm{NPQ}(\mathrm{T})$ was reduced in the H.C. line but was unaltered in the

242 transplastomic lines, in agreement with the observed ФNPQ (Fig. 4C and fig. S18H). Conductance

243 was also reduced in the pNLyc\#2 line and increased in the H.C. and LCe lines (fig. S18F). The

244 rETR was unchanged in the transplastomic pNLyc\#2 and LCe lines but was increase in the H.C.

245 line (fig. S18G). These results suggest that the nuclear H.C. line is the one with the most enhanced

246 photosynthetic efficiency, despite its smaller shoot size.

\section{$L C Y B$-expressing lines show enhanced abiotic stress tolerance and shelf life}

249 The increases in xanthophyll and hormone contents were further assessed, given their functions in 250 photoprotection and stress tolerance, by exposing the transgenic lines to abiotic stress. Leaves of the 251 pNLyc\#2 and H.C. transgenic lines, which had higher xanthophyll content, showed high light 252 tolerance, as measured by the luminescence produced by the accumulation of lipid peroxides (Fig. 253 4D). The LCe line showed no significant increase in high light tolerance (Fig. 4D). In addition, all 254 the transgenic lines showed higher growth rates when exposed to either water deficit or salinity 255 treatments (for 10 and seven days, respectively) when compared to their wild type counterparts 256 (Fig. 4E-F and fig. S19). An extended fruit shelf-life has previously been reported in tomato and 257 other fruit due to enhanced ABA content or to the content of other primary metabolites (e.g., 258 putrescine), so we also examined fruit shelf-life in the transgenic lines. All transgenic lines showed 259 enhanced shelf-life at different time points after harvest when compared to their respective wild types (Fig. 4G and fig. S20). 


\section{DISCUSSION}

263 The tomato is one of the most important fruit and vegetable crops worldwide, but its productivity is

264 affected by several abiotic stresses that have deleterious effects on fruit number and size, as well as

265 on fruit quality (Gerszberg and Hnatuszko-Konka, 2017). In the present study, we have

266 demonstrated that $\angle C Y B$ expression has beneficial effects on tomato plant fitness, stress tolerance,

267 and biomass, regardless of the $L C Y B$ genetic origin, tomato cultivar, or genetic transformation

268 strategy (Table S1). However, the mechanisms by which the introduction of a $L C Y B$ gene

269 modulates plant growth and development, photosynthetic efficiency, and stress tolerance remain

270 unresolved. LCYB catalyzes the conversion of lycopene to $\beta$-carotene, a step previously

271 characterized as a metabolic hot spot in tobacco (Kossler et al., 2021; Moreno et al., 2020). The

272 metabolic hot spot focused on $\beta$-carotene reflects its multiple functions in several molecular and

273 physiological processes (e.g., photosynthesis, oxidative stress). In addition, $\beta$-carotene serve as

274 precursor of xanthophylls (photoprotection), hormones (growth, development, and stress response),

275 and growth regulators (Fig. 5A). Thus, changes in carotenoid content could directly influence

276 photosynthesis, antioxidant properties, and pigment content, while also indirectly influencing

277 hormone and apocarotenoid content (ABA, SLs, $\beta-\mathrm{cc}$ ) and, consequently, plant growth,

278 development, and stress responses (Al-Babili and Bouwmeester, 2015; Nambara and Marion-Poll,

279 2005; Wang et al., 2019).

280 Feedback mechanisms between carotenoids, methylerythritol phosphate (MEP), and ABA

281 pathways, can also influence carotenoid accumulation in maize, rice, Arabidopsis, and tomato (Bai

282 et al., 2009; Beyer et al., 2002; Qin et al., 2007; Romer et al., 2000). Enhanced PSY expression in

283 etiolated Arabidopsis seedlings also resulted in enhanced carotenoid levels via post-translational

284 accumulation of DXS mRNA, which stimulated the supply of MEP substrates (Rodriguez-Villalon

285 et al., 2009b; a). Thus, any alteration in the expression of a carotenogenic gene can impact the

286 expression of other carotenoid genes, as well as key genes from other isoprenoid pathways (e.g.,

$287 D X S, G A 20 o x, C H L$ ), as observed in DcLCYB1 tobacco lines (Moreno et al., 2020). This reflects a

288 close interconnection between the isoprenoid pathways (Fig. 5A) and suggests that any disturbance

289 in the metabolic flux of a particular isoprenoid pathway (e.g., carotenoid pathway) may affect other

290 plastidial isoprenoid-related pathways. Notably, isoprenoids are also the precursors of gibberellins

291 (GAs), brassinosteroids, and cytokinins (CKs), so any disturbance in the isoprenoid flux might

292 influence hormone contents, with subsequent impacts on plant growth, development, and stress

293 tolerance (Gudesblat and Russinova, 2011; Ha et al., 2012; Hedden and Phillips, 2000; Krishna,

294 2003; Schaller et al., 2015; Tran et al., 2007). In fact, the transgenic tomato lines analyzed here are 
evidence of carotenoids as a metabolic hot spot (Fig. 5) because, despite the differences in their genetic background, these tomato lines universally displayed changes in carotenoids, apocarotenoids, and hormone contents (Fig. 2) that resulted in altered growth regulation and biomass partitioning in different tissues (Fig. 1 and figs. S1-4). These changes were furthermore reflected in plant biomass accumulation, resilience to abiotic stresses, and crop productivity (Fig. 1 and Fig. 4D-F).

The hormonal changes and their effects on primary metabolism can explain the changes in biomass accumulation and stress tolerance (Moreno et al., 2021; Moreno et al., 2020; Sheyhakinia et al., 2020; Yoshida et al., 2014). For instance, gibberellins (GAs) control many aspects of growth (e.g., plant height, internode length) and plant development. Bioactive GAs $\left(\mathrm{GA}_{4}\right.$ and $\left.\mathrm{GA}_{1}\right)$ function as key players in plant growth and development in Arabidopsis, tobacco, and rice, with $\mathrm{GA}_{4}$ showing the highest bioactivity (Cowling et al., 1998; Gallego-Giraldo et al., 2008; Talon et al., 1990; Ueguchi-Tanaka et al., 2007). Both the bioactive GAs are produced from $\mathrm{GA}_{12}$ by the non-13hydroxylation $\left(\mathrm{GA}_{4}\right)$ and the early-13-hydroxylation $\left(\mathrm{GA}_{1}\right)$ pathways (Magome et al., 2013). Interestingly, manipulation of GA biosynthetic genes (e.g., COPALYL SYNTHASE, GA3oxidase 1, GA20oxidase 1) in Arabidopsis, tobacco, and rice, showed opposite $\mathrm{GA}_{4}$ and $\mathrm{GA}_{1}$ accumulation patterns (Fleet et al., 2003; Gallego-Giraldo et al., 2008; Magome et al., 2013). In our lines, the longer stems and internode lengths (Fig. 1A, C, J, and fig. S18A, D) in the transplastomic lines suggest an enhanced $\mathrm{GA}_{4}$ content. However, the $\mathrm{GA}_{4}$ level was below the detection limit in the material we profiled in our study, although we detected a reduction in $\mathrm{GA}_{1}$, which could potentially reflect an increase in $\mathrm{GA}_{4}$ in the transplastomic lines. By contrast, the shorter stem and internodes, together with enhanced $\mathrm{GA}_{1}$ content, in the H.C. line suggest a possibly decreased $\mathrm{GA}_{4}$ content (Fig. 1B, J, and fig. S18A, D).

The reduced-growth phenotype is in line with the reduced plant size previously reported in ABAdeficient mutants of tomato (Nitsch et al., 2012). However, a similar ABA reduction in LCe, which shows an opposite phenotype to H.C. (longer stem and internodes), suggests that the interaction between $\mathrm{GA}_{4}$ and $\mathrm{ABA}$ might direct plant height, as previously observed in $D c L C Y B 1$ tobacco lines (Moreno et al., 2020). In addition, reductions in $\beta$-cyclocitral and/or zaxinone in the transplastomic lines (Fig. 2C) suggest that they are not involved in the observed growth phenotype, while reductions in both metabolites might contribute to the smaller growth phenotype observed in the H.C. line (Fig. 1). The enhanced ABA and JA ( $\mathrm{p}=1.1 \mathrm{e}^{-3}$ and $\mathrm{p}=4 \mathrm{e}^{-3}$; Fig. 2B and fig. S9) contents in pNLyc\#2 are likely responsible for its salt and drought tolerance (Fig. 4E, F, and fig. S19), as 
327 previously shown in Arabidopsis and tobacco (Kazan, 2015; Moreno et al., 2021; Moreno et al.,

328 2020; Yoshida et al., 2014).

329 An enhanced ABA content may have caused stomatal closure, as reflected in the observed reduction 330 in stomatal conductance (fig. S18F). This reduction would conceivably impede an enhancement of 331 photosynthetic efficiency (Fig. 4A-C, and fig. S18F-I). By contrast, the H.C. and LCe lines 332 displayed a slightly reduced ABA content and enhanced conductance; however, only the H.C. line 333 showed enhanced photosynthetic efficiency (higher $\mathrm{CO}_{2}$ assimilation, rETR, and ФPSII; Fig. 4A-C, 334 and fig. S18F-I). Although these lines showed reduced ABA content, they both showed enhanced 335 salt and drought tolerance, suggesting the participation of an ABA-independent pathway. In fact, JA/JA-Ile are involved in salt and drought tolerance in Arabidopsis and rice (Hazman et al., 2019; Kazan, 2015). Increases in JA and JA-Ile ( $\mathrm{p}=0.05$ and $\mathrm{p}<1 \mathrm{e}^{-4}$; Fig. $2 \mathbf{B}$ and fig. S9) in the LCe line supported the higher drought and salt tolerance observed in this line. However, the H.C. line showed reductions in ABA and no changes in JA, but a significant increase in IAA ( $p=0.03$; Fig. 2B and fig. S9).

IAA has been reported to enhance salt and drought tolerance in white clover, Arabidopsis, and rice (Shani et al., 2017; Sharma et al., 2013; Shi et al., 2014; Zhang et al., 2020), supporting its enhanced tolerance to these abiotic stresses (Fig. 4E, F, and fig. S19). In addition, several osmoprotectants, which are neutral molecules that help the organisms to persist during severe osmotic stress (Singh et al., 2015), were enhanced in the transgenic lines (Fig. 3A). Increased ABA and JA contents were previously reported to enhance the synthesis of osmoprotectants (e.g., sugars, polyamines) under abiotic stress conditions to counteract harmful effects (Alcazar et al., 2006; Sheyhakinia et al., 2020; Toumi et al., 2010; Wang et al., 2020). In line with this evidence, increases in sugars (raffinose, fructose, G6P, glucose, trehalose), sugar alcohols (myo-inositol, erythritol) and polyamines (putrescine) in leaves can also contribute to enhanced stress tolerance in our transgenic lines (Fig. 3A, Fig. 4E, F, and fig. S19; Table S1).

The increased xanthophyll content in leaves could further enhance photoprotection and therefore impart high light tolerance (pNLyc\#2 and H.C.; Fig. 4D). In the fruit, stronger increases in $\beta$ carotene content caused stronger changes in hormone content, thereby impacting fruit dry matter (up to $67-77 \%$ in semi-controlled and uncontrolled conditions, respectively), size, and number, as well as seed production (Fig. 1, Fig. 2A, B, and fig. S4; Table S1), making the fruit rich in provitamin A and enhancing its nutritional value. Fruit growth is influenced by CKs, Aux, GA, and ABA (Quinet et al., 2019). Transgenic fruit differentially accumulate IAA, iP, ABA, and GA intermediates, suggesting that their interaction may have led to the observed fruit growth 

S10).

Unfortunately, $\mathrm{GA}_{1}$, which was reported to be the most bioactive GA influencing fruit growth (Garcia-Hurtado et al., 2012), was under the detection limit in fruit in our experiments, but its content might explain the large increase in fruit size in the H.C. line. Furthermore, changes in the hormonal network might confer additional advantages to the shoots or fruit. Recently, Diretto et al. showed that the enhanced shelf-life of $L C Y B$-expressing tomato lines was due to increased ABA content and its negative impact on ethylene content (Diretto et al., 2020). Increased ABA content in the pNLyc\#2 and H.C. lines conferred longer fruit shelf-life compared to the wild type (Fig. 4G and fig. S20). However, in the LCe line, which also showed enhanced shelf-life, the ABA content was unchanged, suggesting that shelf-life might be controlled by other factors. Indeed, polyamines (e.g., spermidine, putrescine) are known anti-senescence agents which increase fruit firmness, delay ethylene emission and the climacteric respiratory burst, and induce mechanical stress resistance

373 (Valero et al., 2002). The highest ornithine and putrescine content $(\mathrm{p}<0.05)$ was observed in the 374 LCe line, and this could contribute to the enhanced shelf-life observed in the fruit of this line (Fig.

\section{A; Table S1).}

Accumulation of sugars and derivatives (e.g., raffinose, galactinol, myo-inositol, and trehalose) and amino acids (e.g., Val, Asp, Asn, Thr, Glu, Gln, and Ala) in fruit were reported to confer tolerance to chilling injury and resistance to pathogens and several postharvest stress conditions (Bang et al., 2019; Farcuh et al., 2018; Lauxmann et al., 2014; Luengwilai et al., 2018). Accumulation of these metabolites would be expected to confer valuable post-harvest traits to our tomatoes apart from the enhanced shelf-life and their higher pro-vitamin A content.

The use of transgenic tomato lines with different cultivar and genetic backgrounds allowed us to demonstrate that i) $L C Y B$ overexpression can be used to modulate growth (different biomass partitioning between leaf and fruit) and fruit yield in a crop, and ii) the positive growth regulatory effect conferred by the carrot DcLCYBI gene in tobacco (Moreno et al., 2020) can be also conferred by other LCYBs (e.g., tomato, daffodil, and bacteria) in leaves and/or fruit. However, the different genetic origins of the chosen $L C Y B$ genes also introduced specific changes in each line (Table 1; biotechnological purposes. 
cultivars, transformation methods, and $L C Y B$ genetic origins (Table S1), many similarities can be explained by the modulation of molecular processes, such as carotenoid and hormone accumulations (see above; fig. S21). Despite the observed specific changes in carotenoid, hormone, and metabolite accumulation in leaves and fruit of the transgenic lines (Table 1; Table S2-3 and fig. S21), the similar responses in these lines can be attributed to changes in specific hormones (salt and drought tolerance are most likely conferred by increases in ABA and JA for pNLyc\#2, IAA for H.C., and JA and JA-Ile for LCe; Table 1; fig. S21) and/or metabolites (e.g., putrescine-enhanced shelf-life). However, other observed contrasting phenotypes (e.g., plant height and seed yield) were probably caused by specific interactions between hormones and/or their ratios, as well as the connection between carotenoids and other non-isoprenoid hormones (e.g., IAA), and these remain to be investigated. Nevertheless, modulation of the content of main components of the hormonal network in each transgenic line resulted in enhanced abiotic stress tolerance, extended fruit shelf life, and increased biomass (favoring shoot and/or fruit in the different lines), along with the enhanced nutritional value conferred by the higher $\beta$-carotene content in the fruit (Table 1; fig. S21). All these features are highly desirable traits for crop improvement (especially stress tolerance and higher biomass/yield) considering the worldwide climate change and its consequences for food crop production. This type of bioengineering is a promising strategy that can be exported to cereal crops (e.g., rice) that, in general, do not accumulate high levels of carotenoids but whose yield must be greatly increased by 2050 .

\section{METHODS}

\section{Plant material and growth conditions}

Tomato wild type (S. lycopersicum cvs. IPA6+/lutein, IPA6-/without lutein and isogenic Red Setter/R.S.), transplastomic (pNLyc\#2 and LCe), and nuclear (high carotenoid/H.C.) lines (Apel and Bock, 2009; D'Ambrosio et al., 2004; Wurbs et al., 2007) were raised from seeds germinated on soil. The transgenic lines harbor $L C Y B$ genes from daffodil, tomato, and bacteria (Erwinia uredovora). Two of the selected lines were obtained by plastid DNA transformation (pNLyc\#2 and LCe) and the other line by Agrobacterium-mediated nuclear DNA transformation (H.C.; Table S1). Transplastomic lines expressing the LCYB gene from daffodil or Erwinia uredovora (pNLyc\#2 and LCe, respectively) were generated via plastid transformation using particle bombardment. The homoplasmic state (i.e., the absence of residual copies of the wild-type genome) of 22 plants was assessed by subjecting the transgenic plants to double-resistance tests (spectinomycin and streptomycin, $500 \mathrm{mg} \mathrm{l}^{-1}$ ) on synthetic media and by RFLP analysis (Apel and Bock, 2009; Wurbs 
et al., 2007). Due to the homoplasmic state (meaning that plastid DNA was equally modified in all chloroplasts of the transgenic lines) and to the similar phenotype observed in these lines, we selected one line per genotype (pNLyc\#2 and LCe) to carry out the experiments described in this work. The H.C. nuclear line (plus other six LCYB transgenic lines) was obtained via Agrobacterium transformation. All seven transgenic lines expressing the tomato $L C Y B$ were confirmed by Southern blot experiments and by the intense orange color in their fruit in comparison to the isogenic Red Setter control. In addition, northern blot and qPCR experiments confirmed higher transcript accumulations in the transgenic lines in leaves and fruit than in the isogenic wild type Red Setter control (D'Ambrosio et al., 2004; Giorio et al., 2007). Based on this evidence and the similar phenotype obtained in all nuclear lines, we selected the H.C. line with the highest $\beta$-carotene levels for the experiments in this work.

Wild type and transgenic lines were grown side by side, and randomly allocated, in the greenhouse (semi-controlled conditions) under standard conditions (16 h/8 h day/night regime, 450-800 $\mu \mathrm{mol}$ photons $\mathrm{m}^{-2} \mathrm{~s}^{-1}$ combination of artificial light and sunlight, $24{ }^{\circ} \mathrm{C}$, and $65 \%$ relative humidity). Plant height, leaf and fruit number, internode length, and seed yield were recorded. Fully expanded mature source leaves (the $5^{\text {th }}$ leaf) were harvested from six-week-old wild type and transgenic $L C Y B$ tomato plants $(n=5)$ grown in the greenhouse. Fruits were analyzed as five biological replicates from 16-week-old tomato plants. Each biological replicate consisted of a pool of three different fruits from one individual plant.

\section{Physiological measurements and biomass quantification}

The T5 generation wild type (R.S.) and nuclear transformed (H.C.) and wild type (IPA6+ and IPA6) and T3 transplastomic homoplasmic lines (pNLyc\#2 and LCe) were grown directly on soil. Plants were grown for three weeks in a controlled environment $\left(100-250 \mu \mathrm{mol} \mathrm{m} \mathrm{m}^{-2} \mathrm{~s}^{-1}, 23{ }^{\circ} \mathrm{C}\right)$ and then transferred to fully controlled (plant chamber/530 and $53 \mu \mathrm{mol} \mathrm{m} \mathrm{m}^{-2} \mathrm{~s}^{-1}$ red and white light respectively, $16 / 8 \mathrm{~h}$ photoperiod, $70 \%$ relative humidity and $24{ }^{\circ} \mathrm{C}$ ), semi-controlled (greenhouse/average light intensity: $170-380 \mu \mathrm{mol} \mathrm{m}^{-2} \mathrm{~s}^{-1}$, maximum light intensity: $1200 \mu \mathrm{mol} \mathrm{m}$ ${ }^{2} \mathrm{~s}^{-1}$ and $24{ }^{\circ} \mathrm{C}$ ), and uncontrolled conditions (polytunnel/natural climate conditions during springsummer 2019 in Potsdam, Germany). In each climate condition, plants were grown side by side and they were randomly distributed with at least $50 \mathrm{~cm}$ of space between each other. Physiological parameters, such as plant height and leaf and fruit number, were recorded through development (10 to 60-70 days of growth under the different climate conditions) and/or before performing the 
biomass experiment. Plant biomass for plants grown in fully controlled conditions was assessed in 11-week-old plants (only the biomass of the aerial part, leaf and stem, was recorded). Plant (leaves and stem) and fruit biomass for plants grown under semi-controlled conditions was assessed in two groups of 8- and 16-week-old plants, respectively. The first group was grown for quantification of the leaves and stem $(n=5-6)$, and the second was grown for the assessment of fruit biomass $(n=5)$. Both groups were grown in parallel and harvested at different time points (eight and 16 weeks, respectively). The biomass of plants grown under uncontrolled conditions in the polytunnel was measured in 12-week-old tomato plants. In this case, the leaf, stem and fruit biomass was recorded from the same plants. Briefly, leaves, stem, and fruit were separated and the fresh weight was recorded immediately. Subsequently, the leaves, stem, and fruit were dried at $70{ }^{\circ} \mathrm{C}$ for five days, and the dry weight was recorded. Five (biomass) to ten (plant height) biological replicates were used for each experiment under the different climate conditions. For fruit size quantification, the area of three fully ripened fruit detached from three different greenhouse-grown 16-week-old tomato plants was quantified using ImageJ software.

\section{Photosynthesis measurements}

Wild type and transgenic lines were raised from seeds and grown for three weeks under fully controlled conditions in a phytotron $\left(250 \mu \mathrm{mol}\right.$ photons $\mathrm{m}^{-2} \mathrm{~s}^{-1}, 16 \mathrm{~h} / 8 \mathrm{~h}$ day/night, $22{ }^{\circ} \mathrm{C}$ day/18 ${ }^{\circ} \mathrm{C}$ night, $70 \%$ relative humidity; pots of $7 \mathrm{~cm}$ diameter). The plants were then transferred to the greenhouse $\left(16 \mathrm{~h} / 8 \mathrm{~h}\right.$ day/night regime, $450-800 \mu \mathrm{mol}$ photons $\mathrm{m}^{-2} \mathrm{~s}^{-1}$ combination of artificial and sun light, $24{ }^{\circ} \mathrm{C}, 65 \%$ relative humidity), randomly allocated, and acclimated for four weeks before the photosynthetic measurements (49-day-old plants). Photosynthetic parameters, such as $\mathrm{CO}_{2}$ assimilation, conductance, and relative electron transport rate (rETR), were measured with a Li6400XT portable photosynthesis system equipped with a leaf chamber fluorometer (Li-Cor Inc., Lincoln, NE, USA). The measurements were performed during the mornings on fully expanded leaves under growth light conditions (greenhouse, $450 \mu \mathrm{mol}$ (photons) $\mathrm{m}^{-2} \mathrm{~s}^{-1}$ of PAR), with the amount of blue light set at $10 \%$ of the photosynthetically active photon flux density to optimize stomatal aperture. The reference $\mathrm{CO}_{2}$ concentration was set at $400 \mu \mathrm{mol} \mathrm{CO}_{2} \mathrm{~mol}^{-1}$ air. All measurements were performed using a $2 \mathrm{~cm}^{2}$ leaf chamber maintained with a block temperature of $25^{\circ} \mathrm{C}$ and a flow rate of $300 \mathrm{mmol}$ air $\min ^{-1}$. The rETR was calculated according to the method described in (Krall and Edwards, 1992). In addition, total non-photochemical quenching (NPQT), (ФPSII), (ФNPQ), and (ФNO) were measured in the same plants with a MultiSpec (Photosync) instrument (Kuhlgert et al., 2016; Tietz et al., 2017). All measurements were conducted during the 
early morning (9:00-11:00 am) in the same part of the $7^{\text {th }}$ leaf from seven-week-old plants (growing in $20 \mathrm{~cm}$ diameter pots). Five to 12 plants were used for the measurements.

\section{Water deficit and salinity treatments}

Water deficit and salinity treatments were performed under greenhouse conditions. Tomato seeds were sown and raised under control conditions in a phytotron. After three weeks, the seedlings were transferred to the greenhouse and acclimated for four days. The plants were randomized and placed $30 \mathrm{~cm}$ apart. For water deficit experiments, control plants (wt and transgenic) were watered once per day (50-200 mL per plant, depending of their water requirements), whereas stressed plants were not watered. Plant height and leaf number were recorded before the stress treatment was initiated (day 0) and again at day 10 of the stress conditions. Phenotypes were recorded by photography at the same time points. For salinity stress, plants were watered with $100 \mathrm{~mL}$ of water or $100 \mathrm{~mL}$ salt solution $(\mathrm{NaCl} 200 \mathrm{mM})$ once per day for seven days. Plant height and leaf number were recorded at day 0 before the onset of the stress treatment and seven days later. At day seven, the stress treatment was discontinued and all plants were watered with $100 \mathrm{~mL}$ water for one more week. The plants were photographed again at two weeks after the stress onset (one week of salt treatment and a subsequent week of water only). All tomatoes were grown in $13 \mathrm{~cm}$ diameter pots for the stress experiments in the greenhouse. Five to six biological replicates were used for measurements of control and stress-treated plants.

\section{Photooxidative stress}

Leaf discs $\left(1.2 \mathrm{~cm}\right.$ diameter) were floated on water at $10{ }^{\circ} \mathrm{C}$ and simultaneously exposed for $18 \mathrm{~h}$ to strong white light (photon flux density/PFD, $1200 \mathrm{mmol}$ photons $\mathrm{m}^{-2} \mathrm{~s}^{-1}$ ) produced by an array of light-emitting diodes. The stressed leaf discs were then placed on wet filter paper for measurement of autoluminescence emission after a $2 \mathrm{~h}$ dark adaptation, as previously described (Birtic et al., 2011). The emission signal was imaged with a liquid nitrogen-cooled charge-coupled device (CCD) camera (VersArray 1300B, Roper Scientific), with the sensor operating at a temperature of $-110{ }^{\circ} \mathrm{C}$. The acquisition time was $20 \mathrm{~min}$, and on-CCD $2 \times 2$ binning was used, leading to a resolution of $650 \times 670$ pixels. As previously shown, the imaged signal principally emanates from the slow decomposition of the lipid peroxides that accumulated in the samples during the oxidative stress treatment (Birtic et al., 2011). 
bioRxiv preprint doi: https://doi.org/10.1101/2021.05.05.442770; this version posted May 7, 2021. The copyright holder for this preprint (which

was not certified by peer review) is the author/funder, who has granted bioRxiv a license to display the preprint in perpetuity. It is made available under aCC-BY-NC-ND 4.0 International license.

521

522 Shelf-life experiments

523 Tomato fruits $(n=5)$ were harvested from 16-week-old wild type and transgenic lines and kept for

524 seven weeks at $23^{\circ} \mathrm{C}$ and a relative humidity $\sim 20 \%$. The fruit phenotype was recorded $0,8,16,24$,

52532,40 , and 48 days after detachment from the plant.

526 


\section{Microscopy analysis}

528 Fully ripened tomato fruits were detached from 12-week-old tomato plants for further microscopy

529

530

531

532

533

534

535

536

537

538

539

540

541

542

543

544

545

546

547

548

549

550

551

552

553

554

555 analysis. Lycopene and $\beta$-carotene (Lyc $+\beta$-car) were observed with a Leica DM6000B/SP5 confocal laser-scanning microscope (Leica Microsystems, Wetzlar, Germany), following a previously published protocol (D'Andrea et al., 2014). The Lyc $+\beta$-car signal was visualized using laser excitation of $488 \mathrm{~nm}$ and emission between 400 and $550 \mathrm{~nm}$. The total fluorescence of the generated micrographs was quantified using the ROI function in Fiji software, based on collected data from three different tomato fruits from each line.

\section{HPLC analysis of pigments}

Plastid isoprenoids (chlorophylls, carotenoids, and tocopherols) were extracted and quantified as described previously (Emiliani et al., 2018).

\section{Hormone quantification}

Levels of endogenous phytohormones (cytokinins, auxins, jasmonates, abscisates, gibberellins, and salicylic acid) were determined in five biological replicates of freeze-dried tomato leaves and fruit, according to a modified method described previously (Simura et al., 2018). Briefly, samples containing $1 \mathrm{mg}$ DW of biological material were extracted in an aqueous solution of $50 \%$ acetonitrile $(\mathrm{v} / \mathrm{v})$. A mixture of stable isotope-labeled standards of phytohormones was added to validate the LC-MS/MS method. Crude extracts were loaded onto conditioned Oasis HLB columns (30 mg/1 ml, Waters) and washed with $30 \%$ aqueous acetonitrile. Flow-through fractions containing purified analytes were collected and evaporated to dryness in a vacuum evaporator. The chromatographic separation was performed using an Acquity I class system (Waters, Milford, MA, USA) equipped with an Acquity UPLC ${ }^{\circledR}$ CSH C18 RP column $(150 \times 2.1 \mathrm{~mm}, 1.7 \mu \mathrm{m}$; Waters $)$. The eluted compounds were analyzed using a triple quadrupole mass spectrometer (Xevo ${ }^{\mathrm{TM}} \mathrm{TQ}-\mathrm{XS}$, Waters) equipped with an electrospray ionization source. Data were processed with Target Lynx V4.2 software, and final concentration levels of phytohormones were calculated by isotope dilution (Rittenberg and Foster, 1940). 


\section{Metabolite profile analysis}

557 The methyl tert-butyl ether (MTBE) extraction buffer was prepared and samples extracted as described by Salem et al. (Salem et al., 2016). For metabolites, the chromatograms and mass spectra were evaluated using ChromaTOF 1.0 (Leco, www.leco.com) and TagFinder v.4. (Luedemann et al., 2008) software, respectively. The mass spectra were cross-referenced using the Golm

561 Metabolome database (Kopka et al., 2005). Data are reported following the standards (Dataset S1 562 and S2) suggested by Fernie et al. (Fernie et al., 2011).

\section{Lipid profile analysis}

After MTBE extraction, the lipid-containing fraction was dried, resuspended, and analyzed by LCMS. Samples were run in negative and positive mode (Datasets S3 and S4). The mass spectra were processed with the Refiner MS 7.5 (Genedata) and Xcalibur software.

\section{Statistical and data analyses}

Statistical analysis was performed using GraphPad Prism (version 5.0) or R environment (version 3.5.2 https://www.R-project.org/). Growth and plant productivity were quantified by conducting a set of several experiments. First, growth curves (based on plant height) for all the transgenic lines and their respective wild types were determined for plants grown under fully controlled (plant chamber), semi-controlled (greenhouse), and uncontrolled (polytunnel/ "field" experiment) conditions. Ten plants were used for each environmental condition $(n=10)$. The physiological parameters (plant height, leaf number, fruit number) and plant productivity (fresh and dry matter of leaves, stems, and fruit) were quantified on plants grown under fully controlled $(n=5)$, semicontrolled $(n=5-10)$, and uncontrolled conditions $(n=5-10)$. Fruit fresh and dry matter were quantified for the semi-controlled and uncontrolled conditions. Seed yield was quantified in an independent experiment as the total seed production of 12 transgenic and wild type plants for each genotype. Photosynthetic analysis was performed on plants grown under semi-controlled conditions $(n=5-12)$. Water deficit and salinity stress experiments were performed on three-week-old tomato plants grown under greenhouse conditions $(n=5-6)$. A non-paired two-tailed Student t-test was performed to compare each transgenic line with their respective wild type using GraphPad Prism software. Pigment, metabolite, lipid, and hormone quantifications were performed on five to six tomato plants grown under semi-controlled conditions. Pigments and hormones $(n=5)$ were 
587

588

589

590

591

592

593

594

595

596

597

598

599

600

601

602

603

604

605

606

607

608

609

610

611

612

613

614

615

616

617

analyzed with the unpaired two-tailed Student t-test to compare each transgenic line with their respective wild type using GraphPad Prism software. For metabolomics $(n=5)$, data mining, normalization, clustering, and graphical representation were performed using R Software. For lipid analysis, the output data were normalized to the internal standard and the amount of dry sample used for the analysis (Datasets S5 and S6).

For statistical analysis, the MetaboAnalyst webserver was used (Chong et al., 2019; Pang et al., 2020). The data were auto-scaled and normalized. The differences in the distribution of lipid profiles among the transgenic lines were visually explored by principal component analysis (PCA). The supervised partial least squares discriminant analysis (PLS-DA) was used when the separation obtained with PCA was inadequate. Significant differences were determined among the transgenic lines and their respective wild types with the non-parametric Wilcoxon rank-sum test $(n=5)$. The patterns of the lipid species that changed across the groups of samples were further investigated by building heatmaps based on the calculated lipid ratios for the transgenic lines and their respective wild types.

\section{AUTHOR CONTRIBUTIONS}

J.C.M: Conceived the project and the experimental design, performed growth, biomass and yield, salt and drought stress, and fruit shelf-life experiments. J.G.V. and J.C.M.: performed photosynthetic experiments with Li-Cor and Multispec, respectively, and performed metabolite extraction and sample preparation (J.C.M.), and data analysis (J.G.V.). J.M. and S.A.: performed apocarotenoid extraction, sample preparation and data analysis. O.N. and I.P.: performed hormonomics analysis. M.R-C.: performed carotenoid extraction and quantification; S.C. and J.C.M.: performed data analysis from lipidomics and lipid extraction, respectively. M.H.: performed high light stress experiments and lipid peroxide quantification. M.K. and J.C.M.: performed microscopy analysis with assistance of J.C.M. JCM wrote the paper with special input from J.G.V., A.R.F., M.R-C., A.S. and all other coauthors.

\section{ACKNOWLEDGMENTS}

We are grateful to Prof. Dr. Lothar Willmitzer for his support and advice. We thank Prof. Dr. Ralph Bock (Max Planck Institute of Molecular Plant physiology, Golm, Germany) and Dr. Caterina D’Ambrosio (Centro Ricerche Metapontum Agrobios, ALSIA, Italy) for kindly providing the 
618 transplastomic pNLyc\#2 and LCe seeds and the homozygous nuclear High Caro (H.C.) lines,

619 respectively. We thank Dr. Camila Caldana and Anne Michaelis for providing the GC facility and 620 running the GC samples, respectively, and Maria Rosa Rodriguez-Goberna for technical support 621 related with pigment analysis (supported by grant BIO2017-84041-P from the Spanish AEI). In 622 addition, we thank Hana Martínková and Petra Amakorová for their help with phytohormone 623 analyses. The hormonomics work was funded by the Ministry of Education, Youth and Sports of the 624 Czech Republic (European Regional Development Fund-Project "Plants as a tool for sustainable 625 global development" No. CZ.02.1.01/0.0/0.0/16_019/0000827), and the Internal Grant Agency of 626 Palacký University (IGA_PrF_2021_011).

627

628 DECLARATION OF INETERESTS

629 The authors declare no competing interests.

630 


\section{Figure legends}

632 Fig. 1. Tomato plant yield under semi-controlled conditions in the greenhouse. (A-F)

633 Nine-week-old wild type (IPA6+, R.S., and IPA6-) and transgenic tomato lines (pNLyc\#2, H.C.,

634 and LCe) grown under greenhouse conditions. (G-I) Tomato fruits from 16-week-old wild type and

635 transgenic tomato lines grown under greenhouse conditions (top view). (J-O) Plant height, leaf and

636 fruit number, and dry weight biomass (leaf, stem, and fruit) of wild type and transgenic tomato

637 lines. (P-R) Seed yield of wild type and transgenic tomato lines grown under greenhouse

638 conditions. Seed production was measured as the total weight of seeds produced by 12 independent

639 tomato plants of each genotype. Five to 10 biological replicates were used (J-O). Unpaired two-

640 tailed Student t-test was performed to compare transgenic lines with the wild type. wt: wild type;

641 R.S.: Red Setter; H.C.: high carotene; LCe: lycopene $\beta$-cyclase from Erwinia. Scale bar: $10 \mathrm{~cm}$.

Fig. 2. Carotenoid and hormone metabolism in leaf and fruit of $L C Y B$-expressing tomato

643 lines. (A) Carotenoid pathway (left) and carotenoid composition (right) in leaves and fruits of wild

644 type (IPA6+, R.S., and IPA6-) and LCYB transgenic lines (pNLyc\#2, H.C., and LCe) grown in the

645 greenhouse. (B) Hormone profile in leaves and fruits of wild type and transgenic LCYB lines (see

646 figs. S9-10). (C) Quantification of apocarotenoids with conserved growth-promoting properties ( $\beta$ -

647 cyclocitral/ $\beta$-cc and zaxinone/Zax) in leaves and fruits (see figs. S11-15). Leaf samples were

648 collected from the $5^{\text {th }}$ leaf of each of the five biological replicates used per line (six-week-old

649 plants). Fully ripened fruits were collected from 16-week-old tomato plants (from five different

650 biological replicates, each biological replicate comprising a pool of 3 fruits). Unpaired two-tailed

651 Student t-test was performed to compare transgenic lines with the wild type. In A, *: p < 0.05, **: p

$652<0.005 * * *:$ p < 0.0005; in B, *: p < 0.05. wt: wild type; R.S.: Red Setter; H.C.: high carotene;

653 LCe: lycopene $\beta$-cyclase from Erwinia; LOD: limit of detection; F.C.: fold change. Viol:

654 violaxanthin; car: carotene; Zea: zeaxanthin; Neo: neoxanthin; Lyc: lycopene; Phyt: phytoene; Lut:

655 lutein. ABA: abscisic acid; PA: phaseic acid; IAA: indole acetic acid; iP: isopentenyladenine; GA:

656 gibberellins; SA: salicylic acid; JA: Jasmonic acid; Ile: isoleucine; Eth.: ethylene; isop.: isoprenoids.

657 PSY: phytoene synthase; PDS: phytoene desaturase; ZDS: z-carotene desaturase; CRTISO:

658 carotene isomerase; CHX: carotenoid hydroxylase; VDE: violaxanthin de-epoxidase; ZEP:

659 zeaxanthin epoxidase; NXS: neoxanthin synthase.

660 Fig. 3. Metabolic reshaping in leaves and fruits by $L C Y B$ expression in tomato. (A) Primary

661 metabolite profiling in leaves and fruits of wild type (IPA6+, R.S., and IPA6-) and $L C Y B$ transgenic

662 tomato lines (pNLyc\#2, H.C., and LCe). A non-paired two-tailed Student t-test was performed to

663 compare each transgenic line with their wild type $(\mathrm{p}<0.05 ; n=5$ biological replicates). (B) Lipid 
664 profile in fruits of $\angle C Y B$ transgenic tomato lines. The lipid profile in leaves is reported; however, no 665 significant changes were observed (fig. S17). Wilcoxon's test was performed to compare transgenic 666 lines with their wild types ( $\mathrm{p}<0.05 ; n=5$ biological replicates). Changes are shown as the $\log 2$ fold 667 change between the transgenic lines and their respective wild type controls (for more details see fig. 668 S16-17). Asterisks represent significant changes. OG: oxoglutarate; orn: ornithine; GABA: gamma 669 aminobutyric acid; suc: sucrose; fru: fructose; glc: glucose; G6P: glucose-6-phosphate; Fru6P: 670 fructose-6-phosphate; OAS: o-acetylserine; glucar. lac: glucarate-1,4-lactone; DAG: diacylglycerol;

671 DGDG: di-galactosyldiacylglycerol; MGDG: mono-galactosyldiacylglycerol; PC:

672 phosphatidylcholine; PE: phosphatidylethanolamine; PG: phosphatidylglycerol; PS: 673 phosphatidylserine; SQDG: sulfoquinovosyl diacylglycerol; TAG: triacylglycerol.

674 Fig. 4. Photosynthetic parameters, stress tolerance, and shelf life of transgenic LCYB tomato 675 lines. (A) $\mathrm{CO}_{2}$ assimilation. (B) ФPSII. (C) Total non-photochemical quenching (NPQT). $\mathrm{CO}_{2}$ 676 assimilation was measured with a Li-Cor instrument and DPSII and NPQT with a MultiSpec 677 instrument (Photosync). Photosynthetic parameters were measured from leaves of seven-week-old 678 wild type (IPA6+, R.S., and IPA6-) and transgenic (pNLyc\#2, H.C., and LCe) tomato lines grown 679 under greenhouse conditions. All measurements, and especially NPQT, were performed without a 680 dark adaptation period, as described in Tietz et al. (31). Five to 12 biological replicates were used 681 for each photosynthetic measurement. (D) Lipid peroxidation imaging and quantification of tomato 682 leaf discs (six-week-old plants) exposed to a light intensity of $2000 \mu \mathrm{mol}$ photons $\mathrm{m}^{-2} \mathrm{~s}^{-1}$ and a 683 temperature of $7^{\circ} \mathrm{C}$ degrees. (E) Water deficit and salt treatments in three-week-old wild type and 684 transgenic lines ( $n=5-6)$ grown in the greenhouse in $13 \mathrm{~cm}$ pots (see material and methods). Plant 685 height was recorded before and after water deficit and salt treatments. (F) Growth rate (plant height) 686 ratio between transgenic lines and their respective wild type controls. Plant height was measured 687 before (0 days) and after stress onset (10 days for water deficit and seven days for salt treatments) 688 and the growth rate was calculated under control and stress conditions. (G) Tomato shelf life in wild 689 type and transgenic tomato fruits. Tomato fruits from wild type and transgenic lines were harvested 690 from 15-week-old tomato plants. Shelf life was recorded at 48 days post-harvest (see fig. S20 for 691 other time points). A non-paired two-tailed Student t-test was performed to compare transgenic 692 lines with the wild type. wt: wild type; R.S.: Red Setter; H.C.: high carotene; LCe: lycopene $\beta$ 693 cyclase from Erwinia.

694 Fig. 5. Proposed model for $L C Y B$-mediated plant fitness enhancement. (A) Schematic 695 representation of isoprenoid pathways connected by the common precursor GGPP. Conversion of 696 lycopene into $\beta$-carotene represents a major key regulatory point in the branching of the carotenoid 
697 pathway. The greater $\beta$-carotene production is used for greater production of xanthophylls 698 (photoprotection) and hormone synthesis (modulation of plant growth, development, and stress 699 tolerance). Feedback regulation between $L C Y B, P S Y$, and DXS might be controlling the production 700 of GGPP and therefore influencing the content of other isoprenoids (e.g., GAs, tocopherols, and 701 chlorophylls). (B) Metabolic and physiological changes in leaves (left side) and fruits (right side) of 702 the high carotene (H.C.) tomato transgenic line showing the influence on yield, stress tolerance, 703 photosynthetic efficiency, pro-vitamin A content, and fruit shelf life (for comparison with 704 transplastomic lines see fig. S21). Increases (red), reductions (blue), no changes (black), or 705 compounds under the detection limit by the hormonomics approach (grey), are shown. Metabolites 706 (e.g., carotenoids, apocarotenoids, hormones, lipids) with different accumulation profiles (increases 707 and decreases in different metabolites) are shown both in red and blue. Put: putrescine; Orn: 708 ornithine; Lut: lutein; $\beta$-car: $\beta$-carotene; Tocs: tocopherols; Chls: chlorophylls; Apocar: 709 apocarotenoids; GAs: gibberellins; Viol: violaxanthin; Zea: zeaxanthin; BRs: brassinosteroids; iP: 710 isopentenyladenine.

711 Table 1. Summary of phenotypic and molecular changes in leaves and fruits of transgenic LCYB712 expressing tomato lines 


\section{REFERENCES}

714

715

716

717

718

719

720

721

722

723

724

725

726

727

728

729

730

731

732

733

734

735

736

737

738

739

740

741

742

743

744

745

746

747

748

749

750

751

752

753

754

755

756

757

758

759

Al-Babili, S., and Bouwmeester, H.J. (2015). Strigolactones, a novel carotenoid-derived plant hormone. Annu Rev Plant Biol 66:161-186. 10.1146/annurev-arplant-043014-114759.

Alcazar, R., Cuevas, J.C., Patron, M., Altabella, T., and Tiburcio, A.F. (2006). Abscisic acid modulates polyamine metabolism under water stress in Arabidopsis thaliana. Physiol Plantarum 128:448-455. 10.1111/j.1399-3054.2006.00780.x.

Apel, W., and Bock, R. (2009). Enhancement of carotenoid biosynthesis in transplastomic tomatoes by induced lycopene-to-provitamin A conversion. Plant Physiol 151:59-66. 10.1104/pp.109.140533.

Bai, L., Kim, E.H., DellaPenna, D., and Brutnell, T.P. (2009). Novel lycopene epsilon cyclase activities in maize revealed through perturbation of carotenoid biosynthesis. Plant J 59:588-599. 10.1111/j.1365-313X.2009.03899.x.

Bang, J., Lim, S., Yi, G., Lee, J.G., and Lee, E.J. (2019). Integrated transcriptomic-metabolomic analysis reveals cellular responses of harvested strawberry fruit subjected to short-term exposure to high levels of carbon dioxide. Postharvest Biol Tec 148:120-131. 10.1016/j.postharvbio.2018.11.003.

Beyer, P., Al-Babili, S., Ye, X., Lucca, P., Schaub, P., Welsch, R., and Potrykus, I. (2002). Golden Rice: introducing the beta-carotene biosynthesis pathway into rice endosperm by genetic engineering to defeat vitamin A deficiency. J Nutr 132:506S-510S. 10.1093/jn/132.3.506S.

Birtic, S., Ksas, B., Genty, B., Mueller, M.J., Triantaphylides, C., and Havaux, M. (2011). Using spontaneous photon emission to image lipid oxidation patterns in plant tissues. Plant J 67:11031115. 10.1111/j.1365-313X.2011.04646.x.

Chong, J., Wishart, D.S., and Xia, J. (2019). Using MetaboAnalyst 4.0 for Comprehensive and Integrative Metabolomics Data Analysis. Curr Protoc Bioinformatics 68:e86. 10.1002/cpbi.86.

Cowling, R.J., Kamiya, Y., Seto, H., and Harberd, N.P. (1998). Gibberellin dose-response regulation of GA4 gene transcript levels in Arabidopsis. Plant Physiol 117:1195-1203. 10.1104/pp.117.4.1195. D'Alessandro, S., Ksas, B., and Havaux, M. (2018). Decoding beta-Cyclocitral-Mediated Retrograde Signaling Reveals the Role of a Detoxification Response in Plant Tolerance to Photooxidative Stress. Plant Cell 30:2495-2511. 10.1105/tpc.18.00578.

D'Alessandro, S., Mizokami, Y., Legeret, B., and Havaux, M. (2019). The Apocarotenoid betaCyclocitric Acid Elicits Drought Tolerance in Plants. iScience 19:461-473. 10.1016/j.isci.2019.08.003.

D'Ambrosio, C., Giorio, G., Marino, I., Merendino, A., Petrozza, A., Salfi, L., Stigliani, A.L., and Cellini, F. (2004). Virtually complete conversion of lycopene into beta-carotene in fruits of tomato plants transformed with the tomato lycopene beta-cyclase (tlcy-b) cDNA. Plant Science 166:207214. 10.1016/j.plantsci.2003.09.015.

D'Andrea, L., Amenos, M., and Rodriguez-Concepcion, M. (2014). Confocal laser scanning microscopy detection of chlorophylls and carotenoids in chloroplasts and chromoplasts of tomato fruit. Methods Mol Biol 1153:227-232. 10.1007/978-1-4939-0606-2_16.

Dickinson, A.J., Lehner, K., Mi, J., Jia, K.P., Mijar, M., Dinneny, J., Al-Babili, S., and Benfey, P.N. (2019). beta-Cyclocitral is a conserved root growth regulator. Proc Natl Acad Sci U S A 116:1056310567. 10.1073/pnas.1821445116.

Ding, F., Wang, M., Zhang, S., and Ai, X. (2016). Changes in SBPase activity influence photosynthetic capacity, growth, and tolerance to chilling stress in transgenic tomato plants. Sci Rep 6:32741. 10.1038/srep32741.

Diretto, G., Frusciante, S., Fabbri, C., Schauer, N., Busta, L., Wang, Z.H., Matas, A.J., Fiore, A., Rose, J.K.C., Fernie, A.R., et al. (2020). Manipulation of beta-carotene levels in tomato fruits 
results in increased ABA content and extended shelf life. Plant Biotechnology Journal 18:11851199. 10.1111/pbi.13283.

Emiliani, J., D'Andrea, L., Ferreyra, M.L.F., Maulion, E., Rodriguez, E., Rodriguez-Concepcion, M., and Casati, P. (2018). A role for beta,beta-xanthophylls in Arabidopsis UV-B photoprotection. J Exp Bot 69:4921-4933. 10.1093/jxb/ery242.

Farcuh, M., Rivero, R.M., Sadka, A., and Blumwald, E. (2018). Ethylene regulation of sugar metabolism in climacteric and non-climacteric plums. Postharvest Biol Tec 139:20-30. 10.1016/j.postharvbio.2018.01.012.

Fernie, A.R., Aharoni, A., Willmitzer, L., Stitt, M., Tohge, T., Kopka, J., Carroll, A.J., Saito, K., Fraser, P.D., and DeLuca, V. (2011). Recommendations for reporting metabolite data. Plant Cell 23:2477-2482. 10.1105/tpc.111.086272.

Fleet, C.M., Yamaguchi, S., Hanada, A., Kawaide, H., David, C.J., Kamiya, Y., and Sun, T.P. (2003). Overexpression of AtCPS and AtKS in Arabidopsis confers increased ent-kaurene production but no increase in bioactive gibberellins. Plant Physiol 132:830-839. 10.1104/pp.103.021725.

Gallego-Giraldo, L., Ubeda-Tomas, S., Gisbert, C., Garcia-Martinez, J.L., Moritz, T., and LopezDiaz, I. (2008). Gibberellin homeostasis in tobacco is regulated by gibberellin metabolism genes with different gibberellin sensitivity. Plant Cell Physiol 49:679-690. 10.1093/pcp/pcn042.

Garcia-Hurtado, N., Carrera, E., Ruiz-Rivero, O., Lopez-Gresa, M.P., Hedden, P., Gong, F., and Garcia-Martinez, J.L. (2012). The characterization of transgenic tomato overexpressing gibberellin 20-oxidase reveals induction of parthenocarpic fruit growth, higher yield, and alteration of the gibberellin biosynthetic pathway. J Exp Bot 63:5803-5813. 10.1093/jxb/ers229.

Garcia-Molina, A., and Leister, D. (2020). Accelerated relaxation of photoprotection impairs biomass accumulation in Arabidopsis. Nat Plants 6:9-12. 10.1038/s41477-019-0572-z.

Gerszberg, A., and Hnatuszko-Konka, K. (2017). Tomato tolerance to abiotic stress: a review of most often engineered target sequences. Plant Growth Regul 83:175-198. 10.1007/s10725-0170251-x.

Giorio, G., Stigliani, A.L., and D'Ambrosio, C. (2007). Agronomic performance and transcriptional analysis of carotenoid biosynthesis in fruits of transgenic HighCaro and control tomato lines under field conditions. Transgenic Res 16:15-28. 10.1007/s11248-006-9025-3.

Gudesblat, G.E., and Russinova, E. (2011). Plants grow on brassinosteroids. Curr Opin Plant Biol 14:530-537. 10.1016/j.pbi.2011.05.004.

Ha, S., Vankova, R., Yamaguchi-Shinozaki, K., Shinozaki, K., and Tran, L.S. (2012). Cytokinins: metabolism and function in plant adaptation to environmental stresses. Trends Plant Sci 17:172179. 10.1016/j.tplants.2011.12.005.

Hazman, M., Suhnel, M., Schafer, S., Zumsteg, J., Lesot, A., Beltran, F., Marquis, V., Herrgott, L., Miesch, L., Riemann, M., et al. (2019). Characterization of Jasmonoyl-Isoleucine (JA-Ile) Hormonal Catabolic Pathways in Rice upon Wounding and Salt Stress. Rice (N Y) 12:45. 10.1186/s12284-0190303-0.

Hedden, P., and Phillips, A.L. (2000). Gibberellin metabolism: new insights revealed by the genes. Trends Plant Sci 5:523-530. 10.1016/s1360-1385(00)01790-8.

Hou, X., Rivers, J., Leon, P., McQuinn, R.P., and Pogson, B.J. (2016). Synthesis and Function of Apocarotenoid Signals in Plants. Trends Plant Sci 21:792-803. 10.1016/j.tplants.2016.06.001.

Hussain, H.A., Men, S., Hussain, S., Chen, Y., Ali, S., Zhang, S., Zhang, K., Li, Y., Xu, Q., Liao, C., et al. (2019). Interactive effects of drought and heat stresses on morpho-physiological attributes, yield, nutrient uptake and oxidative status in maize hybrids. Sci Rep 9:3890. 10.1038/s41598-01940362-7.

Kazan, K. (2015). Diverse roles of jasmonates and ethylene in abiotic stress tolerance. Trends Plant Sci 20:219-229. 10.1016/j.tplants.2015.02.001. 
808

809

810

811

812

813

814

815

816

817

818

819

820

821

822

823

824

825

826

827

828

829

830

831

832

833

834

835

836

837

838

839

840

841

842

843

844

845

846

847

848

849

850

851

852

853

854

855

Kopka, J., Schauer, N., Krueger, S., Birkemeyer, C., Usadel, B., Bergmuller, E., Dormann, P., Weckwerth, W., Gibon, Y., Stitt, M., et al. (2005). GMD@CSB.DB: the Golm Metabolome Database. Bioinformatics 21:1635-1638. 10.1093/bioinformatics/bti236.

Kossler, S., Armarego-Marriott, T., Tarkowska, D., Tureckova, V., Agrawal, S., Mi, J., Perez da Sousa, L., Aurel Schottler, M., Schadach, A., Frohlich, A., et al. (2021). Lycopene $\beta$-cyclase expression influences plant physiology, development and metabolism in tobacco plants. Journal of Experimental Botany 10.1093/jxb/erab029.

Krall, J.P., and Edwards, G.E. (1992). Relationship between Photosystem-li Activity and Co2 Fixation in Leaves. Physiol Plantarum 86:180-187. DOI 10.1111/j.1399-3054.1992.tb01328.x.

Krishna, P. (2003). Brassinosteroid-Mediated Stress Responses. J Plant Growth Regul 22:289-297. 10.1007/s00344-003-0058-z.

Kromdijk, J., Glowacka, K., Leonelli, L., Gabilly, S.T., Iwai, M., Niyogi, K.K., and Long, S.P. (2016). Improving photosynthesis and crop productivity by accelerating recovery from photoprotection. Science 354:857-861. 10.1126/science.aai8878.

Kuhlgert, S., Austic, G., Zegarac, R., Osei-Bonsu, I., Hoh, D., Chilvers, M.I., Roth, M.G., Bi, K., TerAvest, D., Weebadde, P., et al. (2016). MultispeQ Beta: a tool for large-scale plant phenotyping connected to the open PhotosynQ network. R Soc Open Sci 3:160592. 10.1098/rsos.160592.

Lauxmann, M.A., Borsani, J., Osorio, S., Lombardo, V.A., Budde, C.O., Bustamante, C.A., Monti, L.L., Andreo, C.S., Fernie, A.R., Drincovich, M.F., et al. (2014). Deciphering the metabolic pathways influencing heat and cold responses during post-harvest physiology of peach fruit. Plant Cell Environ 37:601-616. 10.1111/pce.12181.

Lopez-Calcagno, P.E., Fisk, S., Brown, K.L., Bull, S.E., South, P.F., and Raines, C.A. (2019). Overexpressing the $\mathrm{H}$-protein of the glycine cleavage system increases biomass yield in glasshouse and field-grown transgenic tobacco plants. Plant Biotechnol J 17:141-151. 10.1111/pbi.12953.

Luedemann, A., Strassburg, K., Erban, A., and Kopka, J. (2008). TagFinder for the quantitative analysis of gas chromatography--mass spectrometry (GC-MS)-based metabolite profiling experiments. Bioinformatics 24:732-737. 10.1093/bioinformatics/btn023.

Luengwilai, K., Beckles, D.M., Roessner, U., Dias, D.A., Lui, V., and Siriphanich, J. (2018). Identification of physiological changes and key metabolites coincident with postharvest internal browning of pineapple (Ananas comosus L.) fruit. Postharvest Biol Tec 137:56-65. 10.1016/j.postharvbio.2017.11.013.

Magome, H., Nomura, T., Hanada, A., Takeda-Kamiya, N., Ohnishi, T., Shinma, Y., Katsumata, T., Kawaide, H., Kamiya, Y., and Yamaguchi, S. (2013). CYP714B1 and CYP714B2 encode gibberellin 13-oxidases that reduce gibberellin activity in rice. Proc Natl Acad Sci U S A 110:1947-1952. 10.1073/pnas.1215788110.

Moreno, J.C., Martinez-Jaime, S., Kosmacz, M., Sokolowska, E.M., Schulz, P., Fischer, A., Luzarowska, U., Havaux, M., and Skirycz, A. (2021). A Multi-OMICs Approach Sheds Light on the Higher Yield Phenotype and Enhanced Abiotic Stress Tolerance in Tobacco Lines Expressing the Carrot lycopene $\beta$-cyclase1 Gene. Frontiers in Plant Science 1210.3389/fpls.2021.624365.

Moreno, J.C., Mi, J., Agrawal, S., Kossler, S., Tureckova, V., Tarkowska, D., Thiele, W., Al-Babili, S., Bock, R., and Schottler, M.A. (2020). Expression of a carotenogenic gene allows faster biomass production by redesigning plant architecture and improving photosynthetic efficiency in tobacco. Plant J 103:1967-1984. 10.1111/tpj.14909.

Nambara, E., and Marion-Poll, A. (2005). Abscisic acid biosynthesis and catabolism. Annu Rev Plant Biol 56:165-185. 10.1146/annurev.arplant.56.032604.144046.

Nitsch, L., Kohlen, W., Oplaat, C., Charnikhova, T., Cristescu, S., Michieli, P., Wolters-Arts, M., Bouwmeester, H., Mariani, C., Vriezen, W.H., et al. (2012). ABA-deficiency results in reduced plant and fruit size in tomato. J Plant Physiol 169:878-883. 10.1016/j.jplph.2012.02.004. 
Niyogi, K.K., and Truong, T.B. (2013). Evolution of flexible non-photochemical quenching mechanisms that regulate light harvesting in oxygenic photosynthesis. Curr Opin Plant Biol 16:307314. 10.1016/j.pbi.2013.03.011.

Ort, D.R., Merchant, S.S., Alric, J., Barkan, A., Blankenship, R.E., Bock, R., Croce, R., Hanson, M.R., Hibberd, J.M., Long, S.P., et al. (2015). Redesigning photosynthesis to sustainably meet global food and bioenergy demand. Proc Natl Acad Sci U S A 112:8529-8536. 10.1073/pnas.1424031112.

Pang, Z., Chong, J., Li, S., and Xia, J. (2020). MetaboAnalystR 3.0: Toward an Optimized Workflow for Global Metabolomics. Metabolites 1010.3390/metabo10050186.

Pareek, A., Dhankher, O.P., and Foyer, C.H. (2020). Mitigating the impact of climate change on plant productivity and ecosystem sustainability. J Exp Bot 71:451-456. 10.1093/jxb/erz518.

Qin, G., Gu, H., Ma, L., Peng, Y., Deng, X.W., Chen, Z., and Qu, L.J. (2007). Disruption of phytoene desaturase gene results in albino and dwarf phenotypes in Arabidopsis by impairing chlorophyll, carotenoid, and gibberellin biosynthesis. Cell Res 17:471-482. 10.1038/cr.2007.40.

Quinet, M., Angosto, T., Yuste-Lisbona, F.J., Blanchard-Gros, R., Bigot, S., Martinez, J.P., and Lutts, S. (2019). Tomato Fruit Development and Metabolism. Front Plant Sci 10:1554. 10.3389/fpls.2019.01554.

Rittenberg, D., and Foster, G.L. (1940). A NEW PROCEDURE FOR QUANTITATIVE ANALYSIS BY ISOTOPE DILUTION, WITH APPLICATION TO THE DETERMINATION OF AMINO ACIDS AND FATTY ACIDS. Journal of Biological Chemistry:737-744.

Rodriguez-Concepcion, M., Avalos, J., Bonet, M.L., Boronat, A., Gomez-Gomez, L., HorneroMendez, D., Limon, M.C., Melendez-Martinez, A.J., Olmedilla-Alonso, B., Palou, A., et al. (2018). A global perspective on carotenoids: Metabolism, biotechnology, and benefits for nutrition and health. Prog Lipid Res 70:62-93. 10.1016/j.plipres.2018.04.004.

Rodriguez-Villalon, A., Gas, E., and Rodriguez-Concepcion, M. (2009a). Phytoene synthase activity controls the biosynthesis of carotenoids and the supply of their metabolic precursors in darkgrown Arabidopsis seedlings. Plant J 60:424-435. 10.1111/j.1365-313X.2009.03966.x.

Rodriguez-Villalon, A., Gas, E., and Rodriguez-Concepcion, M. (2009b). Colors in the dark: a model for the regulation of carotenoid biosynthesis in etioplasts. Plant Signal Behav 4:965-967. 10.4161/psb.4.10.9672.

Romer, S., Fraser, P.D., Kiano, J.W., Shipton, C.A., Misawa, N., Schuch, W., and Bramley, P.M. (2000). Elevation of the provitamin A content of transgenic tomato plants. Nat Biotechnol 18:666669. 10.1038/76523.

Roy, S.J., Negrao, S., and Tester, M. (2014). Salt resistant crop plants. Current Opinion in Biotechnology 26:115-124. 10.1016/j.copbio.2013.12.004.

Salem, M.A., Juppner, J., Bajdzienko, K., and Giavalisco, P. (2016). Protocol: a fast, comprehensive and reproducible one-step extraction method for the rapid preparation of polar and semi-polar metabolites, lipids, proteins, starch and cell wall polymers from a single sample. Plant Methods 12:45. 10.1186/s13007-016-0146-2.

Schaller, G.E., Bishopp, A., and Kieber, J.J. (2015). The yin-yang of hormones: cytokinin and auxin interactions in plant development. Plant Cell 27:44-63. 10.1105/tpc.114.133595.

Shani, E., Salehin, M., Zhang, Y., Sanchez, S.E., Doherty, C., Wang, R., Mangado, C.C., Song, L., Tal, I., Pisanty, O., et al. (2017). Plant Stress Tolerance Requires Auxin-Sensitive Aux/IAA Transcriptional Repressors. Curr Biol 27:437-444. 10.1016/j.cub.2016.12.016.

Sharma, R., Priya, P., and Jain, M. (2013). Modified expression of an auxin-responsive rice CC-type glutaredoxin gene affects multiple abiotic stress responses. Planta 238:871-884. 10.1007/s00425013-1940-y. 
903

904

905

906

907

908

909

910

911

912

913

914

915

916

917

918

919

920

921

922

923

924

925

926

927

928

929

930

931

932

933

934

935

936

937

938

939

940

941

942

943

944

945

946

947

948

949

Sheyhakinia, S., Bamary, Z., Einali, A., and Valizadeh, J. (2020). The induction of salt stress tolerance by jasmonic acid treatment in roselle (Hibiscus sabdariffa L.) seedlings through enhancing antioxidant enzymes activity and metabolic changes. Biologia 75:681-692. 10.2478/s11756-020-00444-8.

Shi, H., Chen, L., Ye, T., Liu, X., Ding, K., and Chan, Z. (2014). Modulation of auxin content in Arabidopsis confers improved drought stress resistance. Plant Physiol Biochem 82:209-217. 10.1016/j.plaphy.2014.06.008.

Simkin, A.J., McAusland, L., Headland, L.R., Lawson, T., and Raines, C.A. (2015). Multigene manipulation of photosynthetic carbon assimilation increases $\mathrm{CO} 2$ fixation and biomass yield in tobacco. J Exp Bot 66:4075-4090. 10.1093/jxb/erv204.

Simkin, A.J., Lopez-Calcagno, P.E., Davey, P.A., Headland, L.R., Lawson, T., Timm, S., Bauwe, H., and Raines, C.A. (2017). Simultaneous stimulation of sedoheptulose 1,7-bisphosphatase, fructose 1,6-bisphophate aldolase and the photorespiratory glycine decarboxylase- $\mathrm{H}$ protein increases $\mathrm{CO} 2$ assimilation, vegetative biomass and seed yield in Arabidopsis. Plant Biotechnol J 15:805-816. 10.1111/pbi.12676.

Simura, J., Antoniadi, I., Siroka, J., Tarkowska, D., Strnad, M., Ljung, K., and Novak, O. (2018). Plant Hormonomics: Multiple Phytohormone Profiling by Targeted Metabolomics. Plant Physiol 177:476-489. 10.1104/pp.18.00293.

Singh, M., Kumar, J., Singh, S., Singh, V.P., and Prasad, S.M. (2015). Roles of osmoprotectants in improving salinity and drought tolerance in plants: a review. Rev Environ Sci Bio 14:407-426. 10.1007/s11157-015-9372-8.

South, P.F., Cavanagh, A.P., Liu, H.W., and Ort, D.R. (2019). Synthetic glycolate metabolism pathways stimulate crop growth and productivity in the field. Science 36310.1126/science.aat9077.

Talon, M., Koornneef, M., and Zeevaart, J.A. (1990). Endogenous gibberellins in Arabidopsis thaliana and possible steps blocked in the biosynthetic pathways of the semidwarf ga4 and ga5 mutants. Proc Natl Acad Sci U S A 87:7983-7987. 10.1073/pnas.87.20.7983.

Tietz, S., Hall, C.C., Cruz, J.A., and Kramer, D.M. (2017). NPQ(T) : a chlorophyll fluorescence parameter for rapid estimation and imaging of non-photochemical quenching of excitons in photosystem-II-associated antenna complexes. Plant Cell Environ 40:1243-1255. 10.1111/pce.12924.

Timm, S., Wittmiss, M., Gamlien, S., Ewald, R., Florian, A., Frank, M., Wirtz, M., Hell, R., Fernie, A.R., and Bauwe, H. (2015). Mitochondrial Dihydrolipoyl Dehydrogenase Activity Shapes Photosynthesis and Photorespiration of Arabidopsis thaliana. Plant Cell 27:1968-1984. 10.1105/tpc.15.00105.

Toumi, I., Moschou, P.N., Paschalidis, K.A., Bouamama, B., Ben Salem-Fnayou, A., Ghorbel, A.W., Mliki, A., and Roubelakis-Angelakis, K.A. (2010). Abscisic acid signals reorientation of polyamine metabolism to orchestrate stress responses via the polyamine exodus pathway in grapevine. J Plant Physiol 167:519-525. 10.1016/j.jplph.2009.10.022.

Tran, L.S., Urao, T., Qin, F., Maruyama, K., Kakimoto, T., Shinozaki, K., and Yamaguchi-Shinozaki, K. (2007). Functional analysis of AHK1/ATHK1 and cytokinin receptor histidine kinases in response to abscisic acid, drought, and salt stress in Arabidopsis. Proc Natl Acad Sci U S A 104:20623-20628. 10.1073/pnas.0706547105.

Ueguchi-Tanaka, M., Nakajima, M., Katoh, E., Ohmiya, H., Asano, K., Saji, S., Hongyu, X., Ashikari, M., Kitano, H., Yamaguchi, I., et al. (2007). Molecular interactions of a soluble gibberellin receptor, GID1, with a rice DELLA protein, SLR1, and gibberellin. Plant Cell 19:2140-2155. 10.1105/tpc.106.043729. 
950 Valero, D., Martinez-Romero, D., and Serrano, M. (2002). The role of polyamines in the 951 improvement of the shelf life of fruit. Trends Food Sci Tech 13:228-234. Pii S0924-2244(02)001349526

953 Doi 10.1016/S0924-2244(02)00134-6.

954 Wang, J., Song, L., Gong, X., Xu, J.F., and Li, M.H. (2020). Functions of Jasmonic Acid in Plant 955 Regulation and Response to Abiotic Stress. Int J Mol Sci 21ARTN 144610.3390/ijms21041446.

956 Wang, J.Y., Haider, I., Jamil, M., Fiorilli, V., Saito, Y., Mi, J., Baz, L., Kountche, B.A., Jia, K.P., Guo, 957 X., et al. (2019). The apocarotenoid metabolite zaxinone regulates growth and strigolactone 958 biosynthesis in rice. Nat Commun 10:810. 10.1038/s41467-019-08461-1.

959 Wurbs, D., Ruf, S., and Bock, R. (2007). Contained metabolic engineering in tomatoes by 960 expression of carotenoid biosynthesis genes from the plastid genome. Plant J 49:276-288. 961 10.1111/j.1365-313X.2006.02960.x.

962 Xu, P., Chukhutsina, V.U., Nawrocki, W.J., Schansker, G., Bielczynski, L.W., Lu, Y., Karcher, D., 963 Bock, R., and Croce, R. (2020). Photosynthesis without beta-carotene. Elife 910.7554/eLife.58984.

964 Xu, Y. (2016). Envirotyping for deciphering environmental impacts on crop plants. Theor Appl 965 Genet 129:653-673. 10.1007/s00122-016-2691-5.

966 Yoshida, T., Mogami, J., and Yamaguchi-Shinozaki, K. (2014). ABA-dependent and ABA967 independent signaling in response to osmotic stress in plants. Curr Opin Plant Biol 21:133-139. 968 10.1016/j.pbi.2014.07.009.

969 Zhang, Y., Li, Y., Hassan, M.J., Li, Z., and Peng, Y. (2020). Indole-3-acetic acid improves drought 970 tolerance of white clover via activating auxin, abscisic acid and jasmonic acid related genes and 971 inhibiting senescence genes. BMC Plant Biol 20:150. 10.1186/s12870-020-02354-y. 
975

976

977

978

979

980

981

982

983

984

985

986

987

988

989

990

991

992

993

994

995

996

997

998

999

\section{Figures}

A
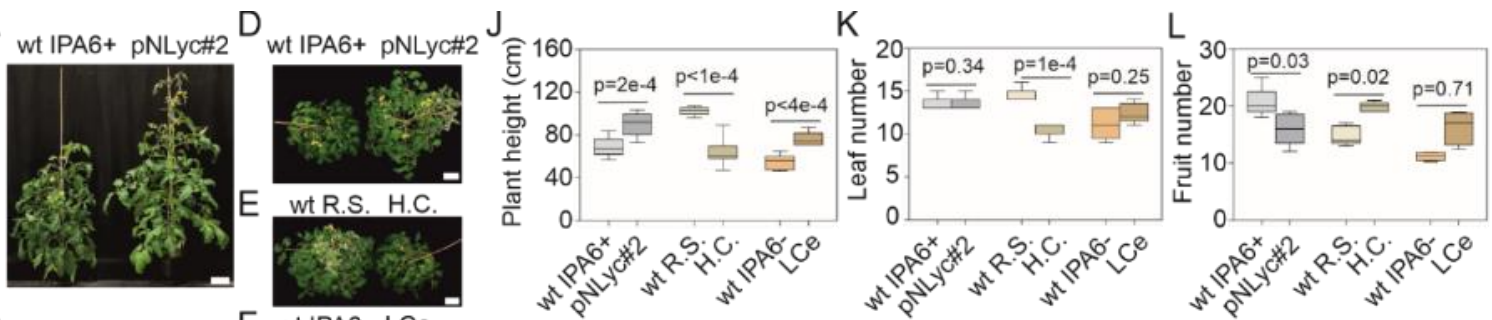

B

F wt IPA6- LCe
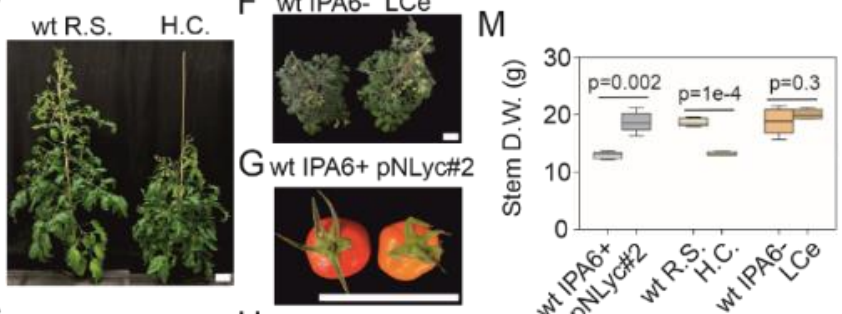

$\mathrm{N}$

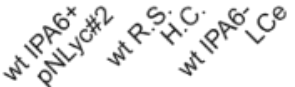

C

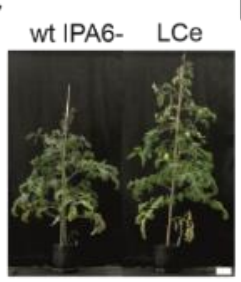

Hurs
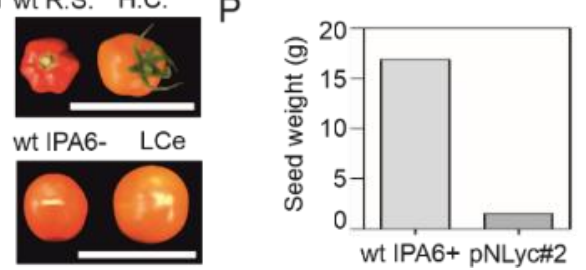

wt IPA6+ pNLyc\#2
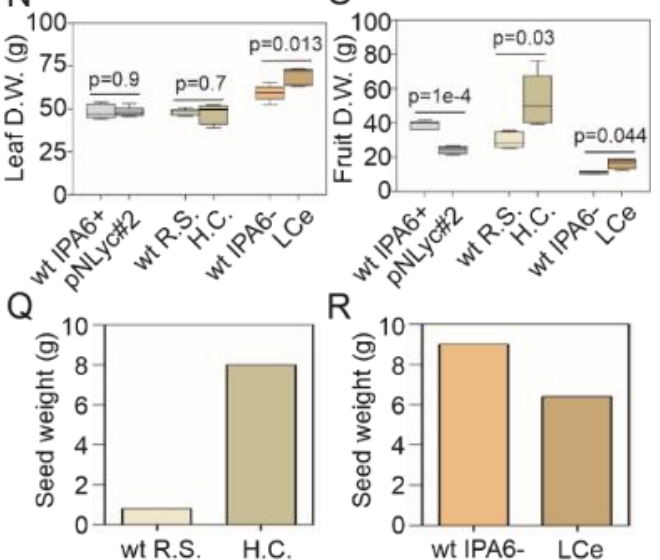

$\mathrm{R}$

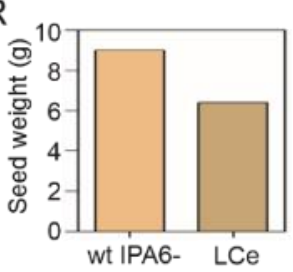

Fig. 1. Tomato plant yield under semi-controlled conditions in the greenhouse. (A-F) Nine-week-old wild type (IPA6+, R.S., and IPA6-) and transgenic tomato lines (pNLyc\#2, H.C., and LCe) grown under greenhouse conditions. (G-I) Tomato fruits from 16-week-old wild type and transgenic tomato lines grown under greenhouse conditions (top view). (J-O) Plant height, leaf and fruit number, and dry weight biomass (leaf, stem, and fruit) of wild type and transgenic tomato lines. (P-R) Seed yield of wild type and transgenic tomato lines grown under greenhouse conditions. Seed production was measured as the total weight of seeds produced by 12 independent tomato plants of each genotype. Five to 10 biological replicates were used (J-O). Unpaired two-tailed Student t-test was performed to compare transgenic lines with the wild type. wt: wild type; R.S.: Red Setter; H.C.: high carotene; LCe: lycopene $\beta$-cyclase from Erwinia. Scale bar: $10 \mathrm{~cm}$. 
1000

1001

1002

1003

1004

1005

1006

1007

1008

1009

1010

1011

1012

1013

1014

1015

1016

1017

1018

1019

1020

1021

1022

1023

1024

1025

1026
A
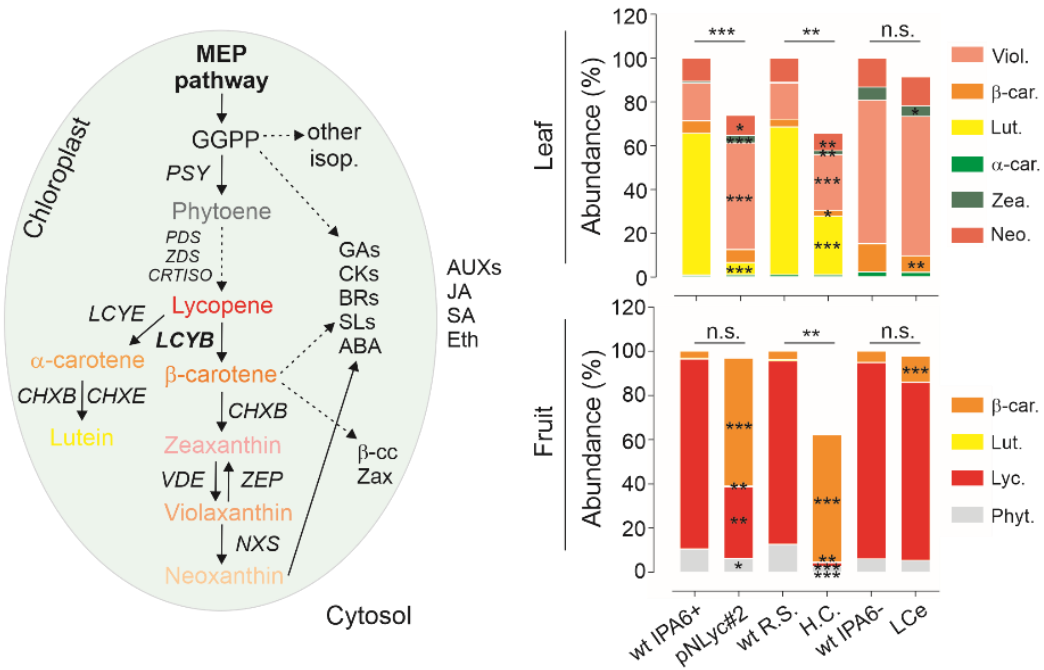

B

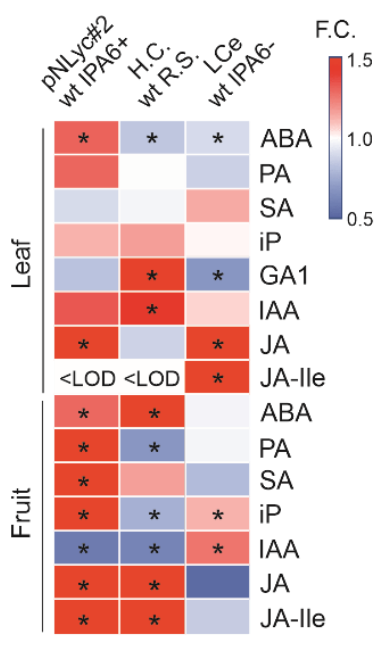

C

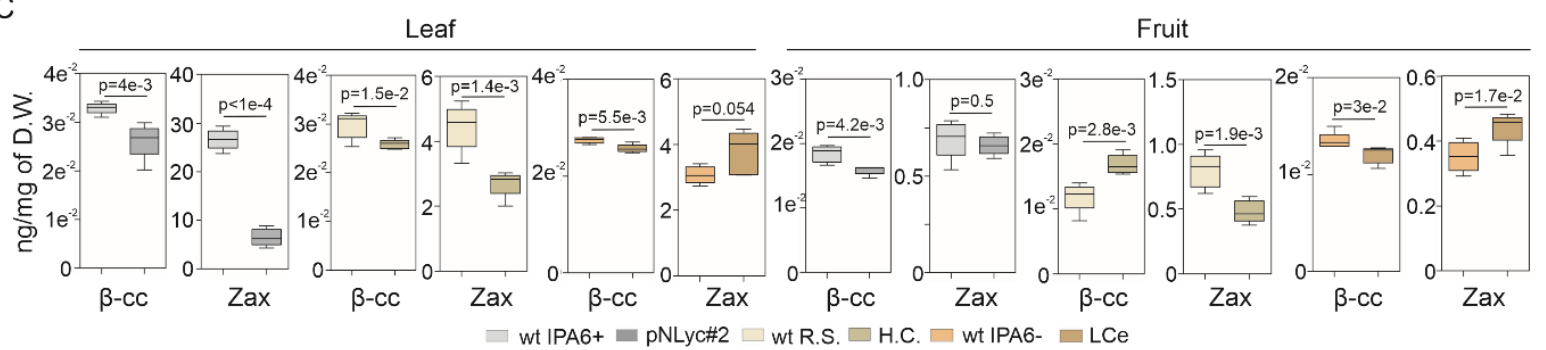

Fig. 2. Carotenoid and hormone metabolism in leaf and fruit of $L C Y B$-expressing tomato lines. (A)

Carotenoid pathway (left) and carotenoid composition (right) in leaves and fruits of wild type (IPA6+, R.S., and IPA6-) and LCYB transgenic lines (pNLyc\#2, H.C., and LCe) grown in the greenhouse. (B) Hormone profile in leaves and fruits of wild type and transgenic LCYB lines (see figs. S9-10). (C) Quantification of apocarotenoids with conserved growth-promoting properties $(\beta$-cyclocitral/ $\beta$-cc and zaxinone/Zax) in leaves and fruits (see figs. S11-15). Leaf samples were collected from the $5^{\text {th }}$ leaf of each of the five biological replicates used per line (six-week-old plants). Fully ripened fruits were collected from 16-week-old tomato plants (from five different biological replicates, each biological replicate comprising a pool of 3 fruits). Unpaired two-tailed Student t-test was performed to compare transgenic lines with the wild type. In $\mathbf{A}, *: \mathrm{p}<0.05$, **: $\mathrm{p}<0.005 * * *: \mathrm{p}<0.0005$; in $\mathbf{B}, *$ : $\mathrm{p}<0.05$. wt: wild type; R.S.: Red Setter; H.C.: high carotene; LCe: lycopene $\beta$-cyclase from Erwinia; LOD: limit of detection; F.C.: fold change. Viol: violaxanthin; car: carotene; Zea: zeaxanthin; Neo: neoxanthin; Lyc: lycopene; Phyt: phytoene; Lut: lutein. ABA: abscisic acid; PA: phaseic acid; IAA: indole acetic acid; iP: isopentenyladenine; GA: gibberellins; SA: salicylic acid; JA: Jasmonic acid; Ile: isoleucine; Eth.: ethylene; isop.: isoprenoids. PSY: phytoene synthase; PDS: phytoene desaturase; ZDS: z-carotene desaturase; CRTISO: carotene isomerase; CHX: carotenoid hydroxylase; VDE: violaxanthin deepoxidase; ZEP: zeaxanthin epoxidase; NXS: neoxanthin synthase. 
1027

1028

1029

1030

1031

1032

1033

1034

1035

1036

1037

1038

1039

1040

1041

1042

1043

1044

1045

1046

1047

1048

1049

1050

1051
A

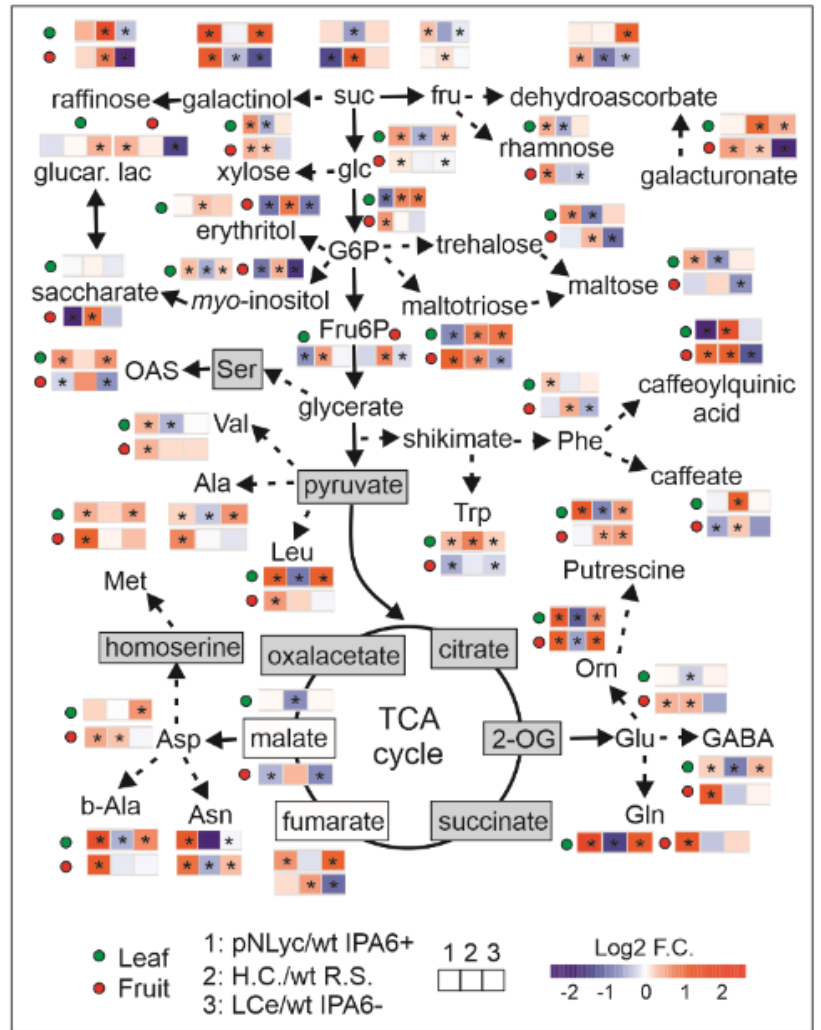

B

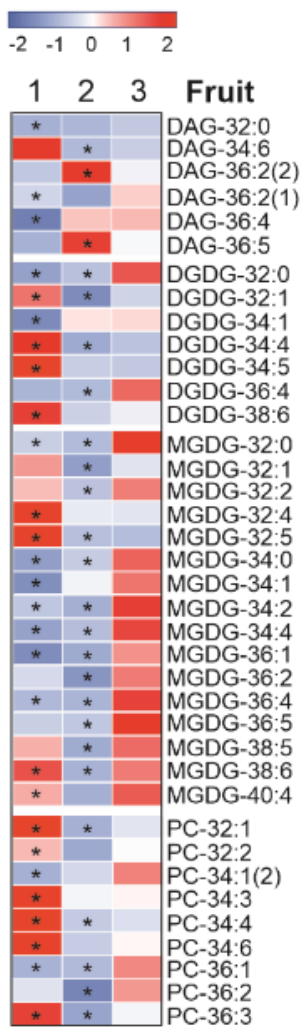

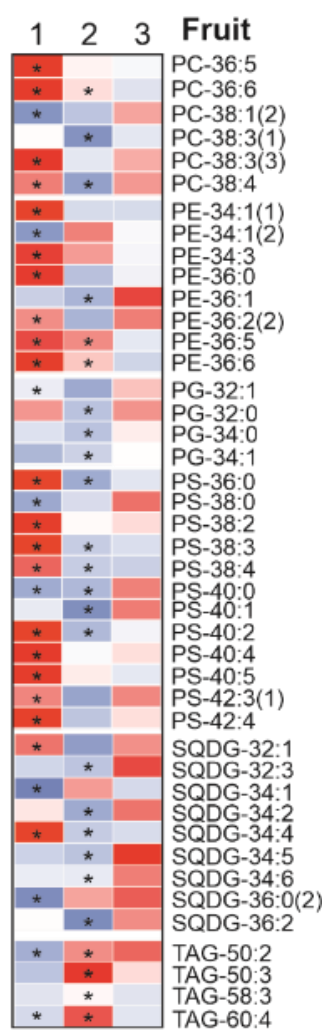

Fig. 3. Metabolic reshaping in leaves and fruits by $L C Y B$ expression in tomato. (A) Primary metabolite profiling in leaves and fruits of wild type (IPA6+, R.S., and IPA6-) and LCYB transgenic tomato lines (pNLyc\#2, H.C., and LCe). A non-paired two-tailed Student t-test was performed to compare each transgenic line with their wild type ( $\mathrm{p}<0.05 ; n=5$ biological replicates). (B) Lipid profile in fruits of $L C Y B$ transgenic tomato lines. The lipid profile in leaves is reported; however, no significant changes were observed (fig. S17). Wilcoxon's test was performed to compare transgenic lines with their wild types ( $\mathrm{p}<0.05 ; n=5$ biological replicates). Changes are shown as the $\log 2$ fold change between the transgenic lines and their respective wild type controls (for more details see fig. S16-17). Asterisks represent significant changes. OG: oxoglutarate; orn: ornithine; GABA: gamma aminobutyric acid; suc: sucrose; fru: fructose; glc: glucose; G6P: glucose-6-phosphate; Fru6P: fructose-6-phosphate; OAS: oacetylserine; glucar. lac: glucarate-1,4-lactone; DAG: diacylglycerol; DGDG: di-galactosyldiacylglycerol; MGDG: mono-galactosyldiacylglycerol; PC: phosphatidylcholine; PE: phosphatidylethanolamine; PG: phosphatidylglycerol; PS: phosphatidylserine; SQDG: sulfoquinovosyl diacylglycerol; TAG: triacylglycerol. 
1054

1055

1056

1057

1058

1059

1060

1061

1062

1063

1064

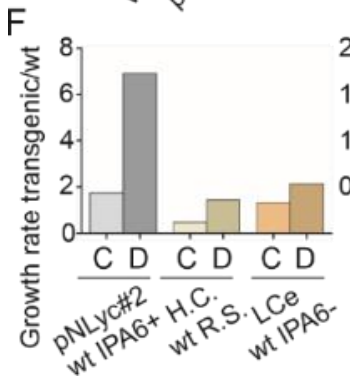

A

C
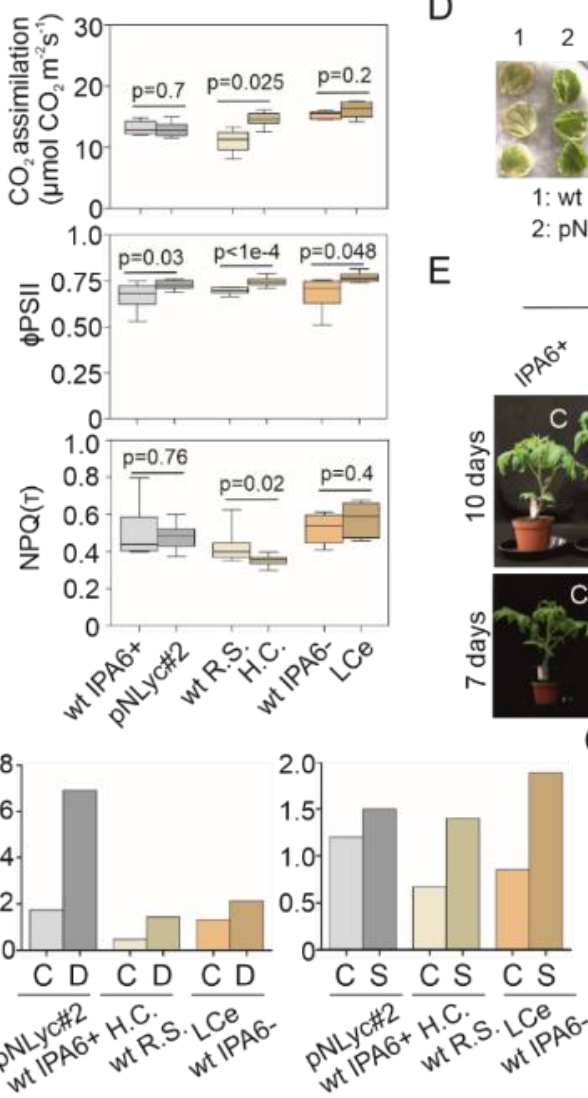

E
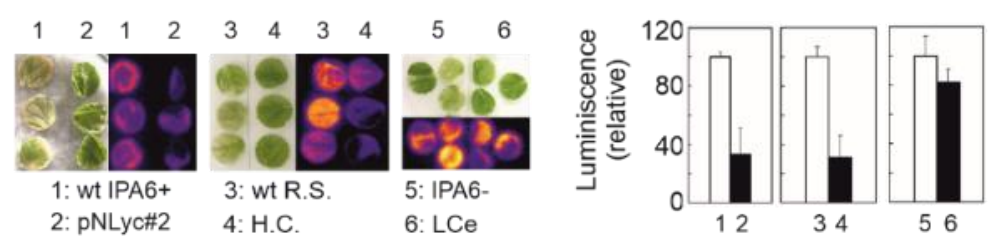

C: control D: drought S: Salt

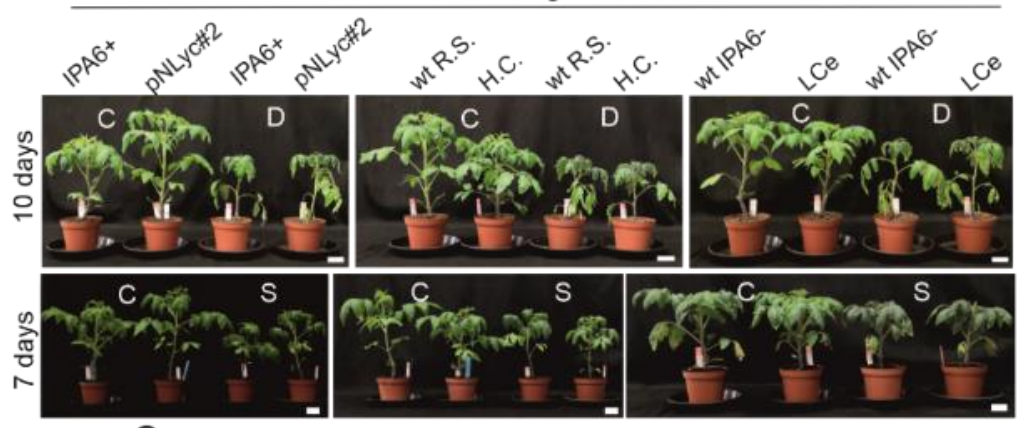

G
1065

1066

1067

1068

1069

1070

1071

1072

1073

1074

1075

1076

1077

1078

1079

1080

Fig. 4. Photosynthetic parameters, stress tolerance, and shelf life of transgenic $L C Y B$ tomato lines. (A) $\mathrm{CO}_{2}$ assimilation. (B) ФPSII. (C) Total non-photochemical quenching (NPQT). $\mathrm{CO}_{2}$ assimilation was measured with a Li-Cor instrument and DPSII and NPQT with a MultiSpec instrument (Photosync). Photosynthetic parameters were measured from leaves of seven-week-old wild type (IPA6+, R.S., and IPA6-) and transgenic (pNLyc\#2, H.C., and LCe) tomato lines grown under greenhouse conditions. All measurements, and especially NPQT, were performed without a dark adaptation period, as described in Tietz et al. (31). Five to 12 biological replicates were used for each photosynthetic measurement. (D) Lipid peroxidation imaging and quantification of tomato leaf discs (six-week-old plants) exposed to a light intensity of $2000 \mu \mathrm{mol}$ photons $\mathrm{m}^{-2} \mathrm{~s}^{-1}$ and a temperature of $7^{\circ} \mathrm{C}$ degrees. (E) Water deficit and salt treatments in three-week-old wild type and transgenic lines $(n=5-6)$ grown in the greenhouse in $13 \mathrm{~cm}$ pots (see material and methods). Plant height was recorded before and after water deficit and salt treatments. (F) Growth rate (plant height) ratio between transgenic lines and their respective wild type controls. Plant height was measured before ( 0 days) and after stress onset (10 days for water deficit and seven days for salt treatments) and the growth rate was calculated under control and stress conditions. (G) Tomato shelf life in wild type and transgenic tomato fruits. Tomato fruits from wild type and transgenic lines were harvested from 15-week-old tomato plants. Shelf life was recorded at 48 days post-harvest (see 
1081 fig. S20 for other time points). A non-paired two-tailed Student t-test was performed to compare 1082 transgenic lines with the wild type. wt: wild type; R.S.: Red Setter; H.C.: high carotene; LCe: lycopene $\beta$ 1083 cyclase from Erwinia.

1084

1085

1086

1087

1088

1089

1090

1091

1092

1093

1094

1095

1096

1097

1098

1099

1100

1101

1102

1103

1104 
A

1107

1108

1109

1110

1111

1112

1113

1114

1115

1116

1117

1118

1119

1120

1121

1122

1123

1124

1125

1126

1127

1128

1129

1130

1131
B pyruvate + G3P
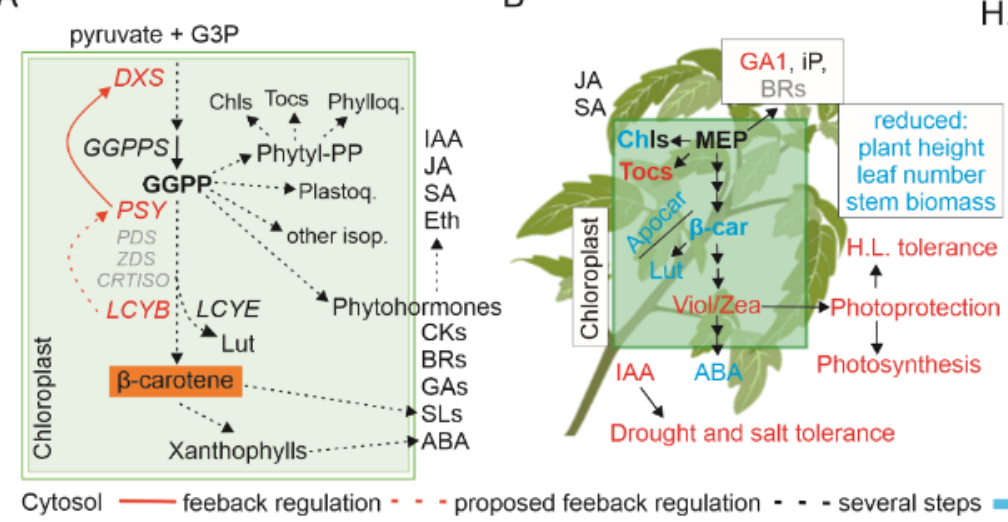

H.C.

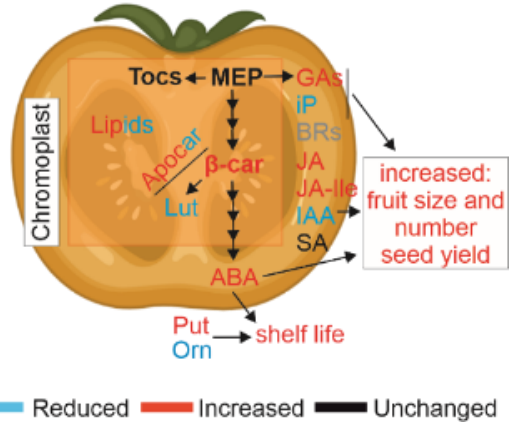

Fig. 5. Proposed model for $L C Y B$-mediated plant fitness enhancement. (A) Schematic representation of isoprenoid pathways connected by the common precursor GGPP. Conversion of lycopene into $\beta$ carotene represents a major key regulatory point in the branching of the carotenoid pathway. The greater $\beta$-carotene production is used for greater production of xanthophylls (photoprotection) and hormone synthesis (modulation of plant growth, development, and stress tolerance). Feedback regulation between $L C Y B, P S Y$, and DXS might be controlling the production of GGPP and therefore influencing the content of other isoprenoids (e.g., GAs, tocopherols, and chlorophylls). (B) Metabolic and physiological changes in leaves (left side) and fruits (right side) of the high carotene (H.C.) tomato transgenic line showing the influence on yield, stress tolerance, photosynthetic efficiency, pro-vitamin A content, and fruit shelf life (for comparison with transplastomic lines see fig. S21). Increases (red), reductions (blue), no changes (black), or compounds under the detection limit by the hormonomics approach (grey), are shown. Metabolites (e.g., carotenoids, apocarotenoids, hormones, lipids) with different accumulation profiles (increases and decreases in different metabolites) are shown both in red and blue. Put: putrescine; Orn: ornithine; Lut: lutein; $\beta$-car: $\beta$-carotene; Tocs: tocopherols; Chls: chlorophylls; Apocar: apocarotenoids; GAs: gibberellins; Viol: violaxanthin; Zea: zeaxanthin; BRs: brassinosteroids; iP: isopentenyladenine. 
Tables

1134 Table 1. Summary of phenotypic and molecular changes in leaves and fruits of transgenic LCYB-

1135 expressing tomato lines.

\begin{tabular}{|c|c|c|c|c|c|c|}
\hline & \multicolumn{3}{|c|}{ Leaf/Shoot } & \multicolumn{3}{|c|}{ Fruit } \\
\hline Phenotype & pNLyc\#2 & H.C. & $\mathrm{LCe}$ & pNLyc\#2 & H.C. & $\mathbf{L C e}$ \\
\hline Plant height & increased & reduced & increased & --- & --- & --- \\
\hline Leaf number & n.c. & reduced & n.c. & --- & --- & --- \\
\hline Internode length & increased & reduced & increased & --- & --- & --- \\
\hline Photosynthesis & n.c. & increased & n.c. & --- & $-\overline{---}$ & --- \\
\hline Days to flowering & increased & reduced & n.c. & --- & --- & --- \\
\hline Fruit number & --- & --- & --- & n.c. & increased & n.c. \\
\hline Fruit size & --- & --- & --- & n.c. & increased & increased \\
\hline Stem D.W. & increased & reduced & n.c. & --- & --- & --- \\
\hline Leaf D.W. & n.c. & n.c. & increased & --- & --- & --- \\
\hline Fruit D.W. & --- & --- & --- & n.c. & increased & n.c. \\
\hline$\beta$-carotene & n.c. & reduced & reduced & increased & increased & increased \\
\hline Xanthophylls & increased & increased & reduced & reduced & reduced & n.d. \\
\hline$\beta$-cyclocitral & reduced & reduced & reduced & increased & increased & reduced \\
\hline Zaxinone & reduced & reduced & n.c. & n.c. & reduced & increased \\
\hline $\mathrm{ABA}$ & increased & reduced & reduced & increased & increased & n.c. \\
\hline SA & n.c. & n.c. & n.c. & increased & n.c. & n.c. \\
\hline iP & n.c. & n.c. & n.c. & increased & reduced & increased \\
\hline $\mathrm{GA}_{1}$ & n.c. & increased & reduced & $<\mathrm{LOD}$ & $<\mathrm{LOD}$ & $<\mathrm{LOD}$ \\
\hline $\mathrm{GA}_{4}$ & $<\mathrm{LOD}$ & $<\mathrm{LOD}$ & $<\mathrm{LOD}$ & $<\mathrm{LOD}$ & $<\mathrm{LOD}$ & $<\mathrm{LOD}$ \\
\hline IAA & n.c. & increased & n.c. & reduced & reduced & increased \\
\hline JA & increased & n.c. & increased & increased & increased & n.c. \\
\hline JA-Ile & $<\mathrm{LOD}$ & $<\mathrm{LOD}$ & increased & increased & increased & n.c. \\
\hline H.L. tolerance & increased & increased & n.c. & --- & --- & --- \\
\hline Drought tolerance & increased & increased & increased & --- & --- & --- \\
\hline Salt tolerance & increased & increased & increased & --- & --- & --- \\
\hline Shelf life & --- & --- & --- & increased & increased & increased \\
\hline
\end{tabular}


bioRxiv preprint doi: https://doi.org/10.1101/2021.05.05.442770; this version posted May 7, 2021. The copyright holder for this preprint (which was not certified by peer review) is the author/funder, who has granted bioRxiv a license to display the preprint in perpetuity. It is made available under aCC-BY-NC-ND 4.0 International license.

1144 


\section{Parsed Citations}

Al-Babili, S., and Bouwmeester, H.J. (2015). Strigolactones, a novel carotenoid-derived plant hormone. Annu Rev Plant Biol 66:161-186. 10.1146/annurev-arplant-043014-114759.

Google Scholar: Author Only Title Only Author and Title

Alcazar, R., Cuevas, J.C., Patron, M., Atabella, T., and Tiburcio, AF. (2006). Abscisic acid modulates polyamine metabolism under water stress in Arabidopsis thaliana. Physiol Plantarum 128:448-455. 10.1111/j.1399-3054.2006.00780.x.

Google Scholar: Author Only Title Only Author and Title

Apel, W., and Bock, R. (2009). Enhancement of carotenoid biosynthesis in transplastomic tomatoes by induced lycopene-to-provitamin Aconversion. Plant Physiol 151:59-66. 10.1104/pp.109.140533.

Google Scholar: Author Only Title Only Author and Title

Bai, L., Kim, E.H., DellaPenna, D., and Brutnell, T.P. (2009). Novel lycopene epsilon cyclase activities in maize revealed through perturbation of carotenoid biosynthesis. Plant J 59:588-599. 10.1111/j.1365-313X.2009.03899.X.

Google Scholar: Author Only Title Only Author and Title

Bang, J., Lim, S., Yi, G., Lee, J.G., and Lee, E.J. (2019). Integrated transcriptomic-metabolomic analysis reveals cellular responses of harvested strawberry fruit subjected to short-term exposure to high levels of carbon dioxide. Postharvest Biol Tec 148:120-131.

10.1016/j.postharvbio.2018.11.003.

Google Scholar: Author Only Title Only Author and Title

Beyer, P., A-Babili, S., Ye, X., Lucca, P., Schaub, P., Welsch, R., and Potrykus, I. (2002). Golden Rice: introducing the beta-carotene biosynthesis pathway into rice endosperm by genetic engineering to defeat vitamin Adeficiency. J Nutr 132:506S-510S.

10.1093/jn/132.3.506S.

Google Scholar: Author Only Title Only Author and Title

Birtic, S., Ksas, B., Genty, B., Mueller, M.J., Triantaphylides, C., and Havaux, M. (2011). Using spontaneous photon emission to image lipid oxidation patterns in plant tissues. Plant J 67:1103-1115. 10.1111/j.1365-313X.2011.04646.x.

Google Scholar: Author Only Title Only Author and Title

Chong, J., Wishart, D.S., and Xia, J. (2019). Using MetaboAnalyst 4.0 for Comprehensive and Integrative Metabolomics Data Analysis. Curr Protoc Bioinformatics 68:e86. 10.1002/cpbi.86.

Google Scholar: Author Only Title Only Author and Title

Cowling, R.J., Kamiya, Y., Seto, H., and Harberd, N.P. (1998). Gibberellin dose-response regulation of GA4 gene transcript levels in Arabidopsis. Plant Physiol 117:1195-1203. 10.1104/pp.117.4.1195.

Google Scholar: Author Only Title Only Author and Title

D'Alessandro, S., Ksas, B., and Havaux, M. (2018). Decoding beta-Cyclocitral-Mediated Retrograde Signaling Reveals the Role of a Detoxification Response in Plant Tolerance to Photooxidative Stress. Plant Cell 30:2495-2511. 10.1105/tpc.18.00578.

Google Scholar: Author Only Title Only Author and Title

D'Alessandro, S., Mizokami, Y., Legeret, B., and Havaux, M. (2019). The Apocarotenoid beta-Cyclocitric Acid Elicits Drought Tolerance in Plants. iScience 19:461-473. 10.1016/j.isci.2019.08.003.

Google Scholar: Author Only Title Only Author and Title

D'Ambrosio, C., Giorio, G., Marino, I., Merendino, A, Petrozza, A, Salfi, L., Stigliani, AL., and Cellini, F. (2004). Virtually complete conversion of lycopene into beta-carotene in fruits of tomato plants transformed with the tomato lycopene beta-cyclase (tlcy-b) cDNA Plant Science 166:207-214. 10.1016/j.plantsci.2003.09.015.

Google Scholar: Author Only Title Only Author and Title

D'Andrea, L., Amenos, M., and Rodriguez-Concepcion, M. (2014). Confocal laser scanning microscopy detection of chlorophylls and carotenoids in chloroplasts and chromoplasts of tomato fruit. Methods Mol Biol 1153:227-232. 10.1007/978-1-4939-0606-2_16.

Google Scholar: Author Only Title Only Author and Title

Dickinson, AJ., Lehner, K., Mi, J., Jia, K.P., Mijar, M., Dinneny, J., A-Babili, S., and Benfey, P.N. (2019). beta-Cyclocitral is a conserved root growth regulator. Proc Natl Acad Sci U S A 116:10563-10567. 10.1073/pnas.1821445116.

Google Scholar: Author Only Title Only Author and Title

Ding, F., Wang, M., Zhang, S., and Ai, X. (2016). Changes in SBPase activity influence photosynthetic capacity, growth, and tolerance to chilling stress in transgenic tomato plants. Sci Rep 6:32741. 10.1038/srep32741.

Google Scholar: Author Only Title Only Author and Title

Diretto, G., Frusciante, S., Fabbri, C., Schauer, N., Busta, L., Wang, ZH., Matas, AJ., Fiore, A, Rose, J.K.C., Fernie, AR., et al. (2020). Manipulation of beta-carotene levels in tomato fruits results in increased ABAcontent and extended shelf life. Plant Biotechnology Journal 18:1185-1199. 10.1111/pbi.13283.

Google Scholar: Author Only Title Only Author and Title

Emiliani, J., D'Andrea, L., Ferreyra, M.L.F., Maulion, E., Rodriguez, E., Rodriguez-Concepcion, M., and Casati, P. (2018). A role for beta,beta-xanthophylls in Arabidopsis UV-B photoprotection. J Exp Bot 69:4921-4933. 10.1093/jxb/ery242. 
Farcuh, M., Rivero, R.M., Sadka, A, and Blumwald, E. (2018). Ethylene regulation of sugar metabolism in climacteric and non-climacteric plums. Postharvest Biol Tec 139:20-30. 10.1016/j.postharvbio.2018.01.012.

Google Scholar: Author Only Title Only Author and Title

Fernie, AR., Aharoni, A, Willmitzer, L., Stitt, M., Tohge, T., Kopka, J., Carroll, AJ., Saito, K., Fraser, P.D., and DeLuca, V. (2011). Recommendations for reporting metabolite data. Plant Cell 23:2477-2482. 10.1105/tpc.111.086272.

Google Scholar: Author Only Title Only Author and Title

Fleet, C.M., Yamaguchi, S., Hanada, A, Kawaide, H., David, C.J., Kamiya, Y., and Sun, T.P. (2003). Overexpression of AtCPS and AtKS in Arabidopsis confers increased ent-kaurene production but no increase in bioactive gibberellins. Plant Physiol 132:830-839.

10.1104/pp.103.021725.

Google Scholar: Author Only Title Only Author and Title

Gallego-Giraldo, L., Ubeda-Tomas, S., Gisbert, C., Garcia-Martinez, J.L., Moritz, T., and Lopez-Diaz, I. (2008). Gibberellin homeostasis in tobacco is regulated by gibberellin metabolism genes with different gibberellin sensitivity. Plant Cell Physiol 49:679-690.

10.1093/pcp/pen042.

Google Scholar: Author Only Title Only Author and Title

Garcia-Hurtado, N., Carrera, E., Ruiz-Rivero, O., Lopez-Gresa, M.P., Hedden, P., Gong, F., and Garcia-Martinez, J.L. (2012). The characterization of transgenic tomato overexpressing gibberellin 20-oxidase reveals induction of parthenocarpic fruit growth, higher yield, and alteration of the gibberellin biosynthetic pathway. J Exp Bot 63:5803-5813. 10.1093/jxb/ers229.

Google Scholar: Author Only Title Only Author and Title

Garcia-Molina, A, and Leister, D. (2020). Accelerated relaxation of photoprotection impairs biomass accumulation in Arabidopsis. Nat Plants 6:9-12. 10.1038/s41477-019-0572-z.

Google Scholar: Author Only Title Only Author and Title

Gerszberg, A, and Hnatuszko-Konka, K. (2017). Tomato tolerance to abiotic stress: a review of most often engineered target sequences. Plant Growth Regul 83:175-198. 10.1007/s10725-017-0251-x.

Google Scholar: Author Only Title Only Author and Title

Giorio, G., Stigliani, AL., and D'Ambrosio, C. (2007). Agronomic performance and transcriptional analysis of carotenoid biosynthesis in fruits of transgenic HighCaro and control tomato lines under field conditions. Transgenic Res 16:15-28. 10.1007/s11248-006-9025-3.

Google Scholar: Author Only Title Only Author and Title

Gudesblat, G.E., and Russinova, E. (2011). Plants grow on brassinosteroids. Curr Opin Plant Biol 14:530-537. 10.1016/j.pbi.2011.05.004. Google Scholar: Author Only Title Only Author and Title

Ha, S., Vankova, R., Yamaguchi-Shinozaki, K., Shinozaki, K., and Tran, L.S. (2012). Cytokinins: metabolism and function in plant adaptation to environmental stresses. Trends Plant Sci 17:172-179. 10.1016/j.tplants.2011.12.005.

Google Scholar: Author Only Title Only Author and Title

Hazman, M., Suhnel, M., Schafer, S., Zumsteg, J., Lesot, A, Beltran, F., Marquis, V., Herrgott, L., Miesch, L., Riemann, M., et al. (2019). Characterization of Jasmonoyl-Isoleucine (JAlle) Hormonal Catabolic Pathways in Rice upon Wounding and Salt Stress. Rice (N Y) 12:45. 10.1186/s12284-019-0303-0.

Google Scholar: Author Only Title Only Author and Title

Hedden, P., and Phillips, AL. (2000). Gibberellin metabolism: new insights revealed by the genes. Trends Plant Sci 5:523-530.

10.1016/s1360-1385(00)01790-8.

Google Scholar: Author Only Title Only Author and Title

Hou, X., Rivers, J., Leon, P., McQuinn, R.P., and Pogson, B.J. (2016). Synthesis and Function of Apocarotenoid Signals in Plants. Trends Plant Sci 21:792-803. 10.1016/j.tplants.2016.06.001.

Google Scholar: Author Only Title Only Author and Title

Hussain, H.A, Men, S., Hussain, S., Chen, Y., Ali, S., Zhang, S., Zhang, K., Li, Y., Xu, Q., Liao, C., et al. (2019). Interactive effects of drought and heat stresses on morpho-physiological attributes, yield, nutrient uptake and oxidative status in maize hybrids. Sci Rep 9:3890. 10.1038/s41598-019-40362-7.

Google Scholar: Author Only Title Only Author and Title

Kazan, K. (2015). Diverse roles of jasmonates and ethylene in abiotic stress tolerance. Trends Plant Sci 20:219-229.

10.1016/j.tplants.2015.02.001.

Google Scholar: Author Only Title Only Author and Title

Kopka, J., Schauer, N., Krueger, S., Birkemeyer, C., Usadel, B., Bergmuller, E., Dormann, P., Weckwerth, W., Gibon, Y., Stitt, M., et al. (2005). GMD@CSB.DB: the Golm Metabolome Database. Bioinformatics 21:1635-1638. 10.1093/bioinformatics/bti236.

Google Scholar: Author Only Title Only Author and Title

Kossler, S., Armarego-Marriott, T., Tarkowska, D., Tureckova, V., Agrawal, S., Mi, J., Perez da Sousa, L., Aurel Schottler, M., Schadach, A, Frohlich, A, et al. (2021). Lycopene $\beta$-cyclase expression influences plant physiology, development and metabolism in tobacco plants. Journal of Experimental Botany 10.1093/jxb/erab029. 
Krall, J.P., and Edwards, G.E. (1992). Relationship between Photosystem-li Activity and Co2 Fixation in Leaves. Physiol Plantarum 86:180-187. DOI 10.1111/j.1399-3054.1992.tb01328.x.

Google Scholar: Author Only Title Only Author and Title

Krishna, P. (2003). Brassinosteroid-Mediated Stress Responses. J Plant Growth Regul 22:289-297. 10.1007/s00344-003-0058-z.

Google Scholar: Author Only Title Only Author and Title

Kromdijk, J., Glowacka, K., Leonelli, L., Gabilly, S.T., Iwai, M., Niyogi, K.K., and Long, S.P. (2016). Improving photosynthesis and crop productivity by accelerating recovery from photoprotection. Science 354:857-861. 10.1126/science.aai8878.

Google Scholar: Author Only Title Only Author and Title

Kuhlgert, S., Austic, G., Zegarac, R., Osei-Bonsu, I., Hoh, D., Chilvers, M.I., Roth, M.G., Bi, K., TerAvest, D., Weebadde, P., et al. (2016). MultispeQ Beta: a tool for large-scale plant phenotyping connected to the open PhotosynQ network. R Soc Open Sci 3:160592.

10.1098/rsos.160592.

Google Scholar: Author Only Title Only Author and Title

Lauxmann, M.A, Borsani, J., Osorio, S., Lombardo, V.A, Budde, C.O., Bustamante, C.A, Monti, L.L., Andreo, C.S., Fernie, AR., Drincovich, M.F., et al. (2014). Deciphering the metabolic pathways influencing heat and cold responses during post-harvest physiology of peach fruit. Plant Cell Environ 37:601-616. 10.1111/pce.12181.

Google Scholar: Author Only Title Only Author and Title

Lopez-Calcagno, P.E., Fisk, S., Brown, K.L., Bull, S.E., South, P.F., and Raines, C.A (2019). Overexpressing the H-protein of the glycine cleavage system increases biomass yield in glasshouse and field-grown transgenic tobacco plants. Plant Biotechnol J 17:141-151.

10.1111/pbi.12953.

Google Scholar: Author Only Title Only Author and Title

Luedemann, A, Strassburg, K., Erban, A, and Kopka, J. (2008). TagFinder for the quantitative analysis of gas chromatography--mass spectrometry (GC-MS)-based metabolite profiling experiments. Bioinformatics 24:732-737. 10.1093/bioinformatics/btn023.

Google Scholar: Author Only Title Only Author and Title

Luengwilai, K., Beckles, D.M., Roessner, U., Dias, D.A, Lui, V., and Siriphanich, J. (2018). Identification of physiological changes and key metabolites coincident with postharvest internal browning of pineapple (Ananas comosus L.) fruit. Postharvest Biol Tec 137:56-65. 10.1016/j.postharvbio.2017.11.013.

Google Scholar: Author Only Title Only Author and Title

Magome, H., Nomura, T., Hanada, A, Takeda-Kamiya, N., Ohnishi, T., Shinma, Y., Katsumata, T., Kawaide, H., Kamiya, Y., and Yamaguchi, S. (2013). CYP714B1 and CYP714B2 encode gibberellin 13-oxidases that reduce gibberellin activity in rice. Proc Natl Acad Sci U S A 110:1947-1952. 10.1073/pnas.1215788110.

Google Scholar: Author Only Title Only Author and Title

Moreno, J.C., Martinez-Jaime, S., Kosmacz, M., Sokolowska, E.M., Schulz, P., Fischer, A, Luzarowska, U., Havaux, M., and Skirycz, A (2021). A Multi-OMICs Approach Sheds Light on the Higher Yield Phenotype and Enhanced Abiotic Stress Tolerance in Tobacco Lines Expressing the Carrot lycopene $\beta$-cyclase1 Gene. Frontiers in Plant Science 1210.3389/fpls.2021.624365.

Google Scholar: Author Only Title Only Author and Title

Moreno, J.C., Mi, J., Agrawal, S., Kossler, S., Tureckova, V., Tarkowska, D., Thiele, W., A-Babili, S., Bock, R., and Schottler, M.A (2020). Expression of a carotenogenic gene allows faster biomass production by redesigning plant architecture and improving photosynthetic efficiency in tobacco. Plant J 103:1967-1984. 10.1111/tpj.14909.

Google Scholar: Author Only Title Only Author and Title

Nambara, E., and Marion-Poll, A (2005). Abscisic acid biosynthesis and catabolism. Annu Rev Plant Biol 56:165-185.

10.1146/annurev.arplant.56.032604.144046.

Google Scholar: Author Only Title Only Author and Title

Nitsch, L., Kohlen, W., Oplaat, C., Charnikhova, T., Cristescu, S., Michieli, P., Wolters-Arts, M., Bouwmeester, H., Mariani, C., Vriezen, W.H., et al. (2012). ABAdeficiency results in reduced plant and fruit size in tomato. J Plant Physiol 169:878-883.

10.1016/j.jplph.2012.02.004.

Google Scholar: Author Only Title Only Author and Title

Niyogi, K.K., and Truong, T.B. (2013). Evolution of flexible non-photochemical quenching mechanisms that regulate light harvesting in oxygenic photosynthesis. Curr Opin Plant Biol 16:307-314. 10.1016/j.pbi.2013.03.011.

Google Scholar: Author Only Title Only Author and Title

Ort, D.R., Merchant, S.S., Aric, J., Barkan, A, Blankenship, R.E., Bock, R., Croce, R., Hanson, M.R., Hibberd, J.M., Long, S.P., et al. (2015). Redesigning photosynthesis to sustainably meet global food and bioenergy demand. Proc Natl Acad Sci U S A112:8529-8536. 10.1073/pnas.1424031112.

Google Scholar: Author Only Title Only Author and Title

Pang, Z, Chong, J., Li, S., and Xia, J. (2020). MetaboAnalystR 3.0: Toward an Optimized Workflow for Global Metabolomics. Metabolites 1010.3390/metabo10050186.

Google Scholar: Author Only Title Only Author and Title 
Pareek, A, Dhankher, O.P., and Foyer, C.H. (2020). Mitigating the impact of climate change on plant productivity and ecosystem sustainability. J Exp Bot 71:451-456. 10.1093/jxb/erz518.

Google Scholar: Author Only Title Only Author and Title

Qin, G., Gu, H., Ma, L., Peng, Y., Deng, X.W., Chen, Z, and Qu, L.J. (2007). Disruption of phytoene desaturase gene results in albino and dwarf phenotypes in Arabidopsis by impairing chlorophyll, carotenoid, and gibberellin biosynthesis. Cell Res 17:471-482.

10.1038/cr.2007.40.

Google Scholar: Author Only Title Only Author and Title

Quinet, M., Angosto, T., Yuste-Lisbona, F.J., Blanchard-Gros, R., Bigot, S., Martinez, J.P., and Lutts, S. (2019). Tomato Fruit

Development and Metabolism. Front Plant Sci 10:1554. 10.3389/fpls.2019.01554.

Google Scholar: Author Only Title Only Author and Title

Rittenberg, D., and Foster, G.L. (1940). ANEW PROCEDURE FOR QUANTITATIVE ANALYSIS BY ISOTOPE DILUTION, WTH APPLICATION TO THE DETERMINATION OF AMINO ACIDS AND FATTY ACIDS. Journal of Biological Chemistry:737-744.

Google Scholar: Author Only Title Only Author and Title

Rodriguez-Concepcion, M., Avalos, J., Bonet, M.L., Boronat, A, Gomez-Gomez, L., Hornero-Mendez, D., Limon, M.C., MelendezMartinez, AJ., Olmedilla-Alonso, B., Palou, A, et al. (2018). Aglobal perspective on carotenoids: Metabolism, biotechnology, and benefits for nutrition and health. Prog Lipid Res 70:62-93. 10.1016/j.plipres.2018.04.004.

Google Scholar: Author Only Title Only Author and Title

Rodriguez-Villalon, A, Gas, E., and Rodriguez-Concepcion, M. (2009a). Phytoene synthase activity controls the biosynthesis of carotenoids and the supply of their metabolic precursors in dark-grown Arabidopsis seedlings. Plant J 60:424-435. 10.1111/j.1365313X.2009.03966.X.

Google Scholar: Author Only Title Only Author and Title

Rodriguez-Villalon, A, Gas, E., and Rodriguez-Concepcion, M. (2009b). Colors in the dark: a model for the regulation of carotenoid biosynthesis in etioplasts. Plant Signal Behav 4:965-967. 10.4161/psb.4.10.9672.

Google Scholar: Author Only Title Only Author and Title

Romer, S., Fraser, P.D., Kiano, J.W., Shipton, C.A, Misawa, N., Schuch, W., and Bramley, P.M. (2000). Elevation of the provitamin A content of transgenic tomato plants. Nat Biotechnol 18:666-669. 10.1038/76523.

Google Scholar: Author Only Title Only Author and Title

Roy, S.J., Negrao, S., and Tester, M. (2014). Salt resistant crop plants. Current Opinion in Biotechnology 26:115-124.

10.1016/j.copbio.2013.12.004.

Google Scholar: Author Only Title Only Author and Title

Salem, M.A, Juppner, J., Bajdzienko, K., and Giavalisco, P. (2016). Protocol: a fast, comprehensive and reproducible one-step extraction method for the rapid preparation of polar and semi-polar metabolites, lipids, proteins, starch and cell wall polymers from a single sample. Plant Methods 12:45. 10.1186/s13007-016-0146-2.

Google Scholar: Author Only Title Only Author and Title

Schaller, G.E., Bishopp, A, and Kieber, J.J. (2015). The yin-yang of hormones: cytokinin and auxin interactions in plant development. Plant Cell 27:44-63. 10.1105/tpc.114.133595.

Google Scholar: Author Only Title Only Author and Title

Shani, E., Salehin, M., Zhang, Y., Sanchez, S.E., Doherty, C., Wang, R., Mangado, C.C., Song, L., Tal, I., Pisanty, O., et al. (2017). Plant Stress Tolerance Requires Auxin-Sensitive Aux/IAA Transcriptional Repressors. Curr Biol 27:437-444. 10.1016/j.cub.2016.12.016.

Google Scholar: Author Only Title Only Author and Title

Sharma, R., Priya, P., and Jain, M. (2013). Modified expression of an auxin-responsive rice CC-type glutaredoxin gene affects multiple abiotic stress responses. Planta 238:871-884. 10.1007/s00425-013-1940-y.

Google Scholar: Author Only Title Only Author and Title

Sheyhakinia, S., Bamary, Z, Einali, A, and Valizadeh, J. (2020). The induction of salt stress tolerance by jasmonic acid treatment in roselle (Hibiscus sabdariffa L.) seedlings through enhancing antioxidant enzymes activity and metabolic changes. Biologia 75:681-692. 10.2478/s11756-020-00444-8.

Google Scholar: Author Only Title Only Author and Title

Shi, H., Chen, L., Ye, T., Liu, X., Ding, K., and Chan, Z (2014). Modulation of auxin content in Arabidopsis confers improved drought stress resistance. Plant Physiol Biochem 82:209-217. 10.1016/j.plaphy.2014.06.008.

Google Scholar: Author Only Title Only Author and Title

Simkin, AJ., McAusland, L., Headland, L.R., Lawson, T., and Raines, C.A (2015). Multigene manipulation of photosynthetic carbon assimilation increases CO2 fixation and biomass yield in tobacco. J Exp Bot 66:4075-4090. 10.1093/jxb/erv204.

Google Scholar: Author Only Title Only Author and Title

Simkin, AJ., Lopez-Calcagno, P.E., Davey, P.A, Headland, L.R., Lawson, T., Timm, S., Bauwe, H., and Raines, C.A (2017). Simultaneous stimulation of sedoheptulose 1,7-bisphosphatase, fructose 1,6-bisphophate aldolase and the photorespiratory glycine decarboxylase-H protein increases CO2 assimilation, vegetative biomass and seed yield in Arabidopsis. Plant Biotechnol J 15:805-816.

10.1111/pbi.12676. 

was not certified by peer review) is the author/funder, who has granted bioRxiv a license to display the preprint in perpetuity. It is made Google Scholar: Author Only Title Only Author and Title available under aCC-BY-NC-ND 4.0 International license.

Simura, J., Antoniadi, I., Siroka, J., Tarkowska, D., Strnad, M., Ljung, K., and Novak, O. (2018). Plant Hormonomics: Multiple Phytohormone Profiling by Targeted Metabolomics. Plant Physiol 177:476-489. 10.1104/pp.18.00293.

Google Scholar: Author Only Title Only Author and Title

Singh, M., Kumar, J., Singh, S., Singh, V.P., and Prasad, S.M. (2015). Roles of osmoprotectants in improving salinity and drought tolerance in plants: a review. Rev Environ Sci Bio 14:407-426. 10.1007/s11157-015-9372-8.

Google Scholar: Author Only Title Only Author and Title

South, P.F., Cavanagh, AP., Liu, H.W., and Ort, D.R. (2019). Synthetic glycolate metabolism pathways stimulate crop growth and productivity in the field. Science $36310.1126 /$ science.aat9077.

Google Scholar: Author Only Title Only Author and Title

Talon, M., Koornneef, M., and Zeevaart, J.A (1990). Endogenous gibberellins in Arabidopsis thaliana and possible steps blocked in the biosynthetic pathways of the semidwarf ga4 and ga5 mutants. Proc Natl Acad Sci U S A 87:7983-7987. 10.1073/pnas.87.20.7983.

Google Scholar: Author Only Title Only Author and Title

Tietz, S., Hall, C.C., Cruz, J.A, and Kramer, D.M. (2017). NPQ(T) : a chlorophyll fluorescence parameter for rapid estimation and imaging of non-photochemical quenching of excitons in photosystem-ll-associated antenna complexes. Plant Cell Environ 40:1243-1255.

10.1111/pce.12924.

Google Scholar: Author Only Title Only Author and Title

Timm, S., Wittmiss, M., Gamlien, S., Ewald, R., Florian, A, Frank, M., Wirtz, M., Hell, R., Fernie, AR., and Bauwe, H. (2015). Mitochondrial Dihydrolipoyl Dehydrogenase Activity Shapes Photosynthesis and Photorespiration of Arabidopsis thaliana. Plant Cell 27:1968-1984.

10.1105/tpc.15.00105.

Google Scholar: Author Only Title Only Author and Title

Toumi, I., Moschou, P.N., Paschalidis, K.A, Bouamama, B., Ben Salem-Fnayou, A, Ghorbel, AW., Mliki, A, and Roubelakis-Angelakis, K.A (2010). Abscisic acid signals reorientation of polyamine metabolism to orchestrate stress responses via the polyamine exodus pathway in grapevine. J Plant Physiol 167:519-525. 10.1016/j.jplph.2009.10.022.

Google Scholar: Author Only Title Only Author and Title

Tran, L.S., Urao, T., Qin, F., Maruyama, K., Kakimoto, T., Shinozaki, K., and Yamaguchi-Shinozaki, K. (2007). Functional analysis of AHK1/ATHK1 and cytokinin receptor histidine kinases in response to abscisic acid, drought, and salt stress in Arabidopsis. Proc Natl Acad Sci U S A 104:20623-20628. 10.1073/pnas.0706547105.

Google Scholar: Author Only Title Only Author and Title

Ueguchi-Tanaka, M., Nakajima, M., Katoh, E., Ohmiya, H., Asano, K., Saji, S., Hongyu, X., Ashikari, M., Kitano, H., Yamaguchi, I., et al. (2007). Molecular interactions of a soluble gibberellin receptor, GID1, with a rice DELLA protein, SLR1, and gibberellin. Plant Cell

19:2140-2155. 10.1105/tpc.106.043729.

Google Scholar: Author Only Title Only Author and Title

Valero, D., Martinez-Romero, D., and Serrano, M. (2002). The role of polyamines in the improvement of the shelf life of fruit. Trends Food Sci Tech 13:228-234. Pii S0924-2244(02)00134-6

Google Scholar: Author Only Title Only Author and Title

Doi 10.1016/S0924-2244(02)00134-6.

Wang, J., Song, L., Gong, X., Xu, J.F., and Li, M.H. (2020). Functions of Jasmonic Acid in Plant Regulation and Response to Abiotic Stress. Int J Mol Sci 21ARTN 144610.3390/ijms21041446.

Google Scholar: Author Only Title Only Author and Title

Wang, J.Y., Haider, I., Jamil, M., Fiorilli, V., Saito, Y., Mi, J., Baz, L., Kountche, B.A, Jia, K.P., Guo, X., et al. (2019). The apocarotenoid metabolite zaxinone regulates growth and strigolactone biosynthesis in rice. Nat Commun 10:810. 10.1038/s41467-019-08461-1.

Google Scholar: Author Only Title Only Author and Title

Wurbs, D., Ruf, S., and Bock, R. (2007). Contained metabolic engineering in tomatoes by expression of carotenoid biosynthesis genes from the plastid genome. Plant J 49:276-288. 10.1111/j.1365-313X.2006.02960.x.

Google Scholar: Author Only Title Only Author and Title

Xu, P., Chukhutsina, V.U., Nawrocki, W.J., Schansker, G., Bielczynski, L.W., Lu, Y., Karcher, D., Bock, R., and Croce, R. (2020). Photosynthesis without beta-carotene. Elife 910.7554/eLife.58984.

Google Scholar: Author Only Title Only Author and Title

Xu, Y. (2016). Envirotyping for deciphering environmental impacts on crop plants. Theor Appl Genet 129:653-673. 10.1007/s00122-0162691-5.

Google Scholar: Author Only Title Only Author and Title

Yoshida, T., Mogami, J., and Yamaguchi-Shinozaki, K. (2014). ABA-dependent and ABA-independent signaling in response to osmotic stress in plants. Curr Opin Plant Biol 21:133-139. 10.1016/j.pbi.2014.07.009.

Google Scholar: Author Only Title Only Author and Title

Zhang, Y., Li, Y., Hassan, M.J., Li, Z, and Peng, Y. (2020). Indole-3-acetic acid improves drought tolerance of white clover via activating 
bioRxiv preprint doi: https://doi.org/10.1101/2021.05.05.442770; this version posted May 7, 2021. The copyright holder for this preprint (which was not certified by peer review) is the author/funder, who has granted bioRxiv a license to display the preprint in perpetuity. It is made

auxin, abscisic acid and jasmonic acid related genes and inhibiting senescence genes. BMC Plant Biol 20:150. 10.1186/s12870-02002354-y.

Google Scholar: Author Only Title Only Author and Title 